\title{
The poset of positive roots and its relatives
}

\author{
Dmitri I. Panyushev
}

Received: December 20, 2004 / Revised: June 14, 2005 / Accepted: July 5, 2005

(C) Springer Science + Business Media, Inc. 2006

\begin{abstract}
Let $\Delta$ be a root system with a subset of positive roots, $\Delta^{+}$. We consider edges of the Hasse diagrams of some posets associated with $\Delta^{+}$. For each edge one naturally defines its type, and we study the partition of the set of edges into types. For $\Delta^{+}$, the type is a simple root, and for the posets of ad-nilpotent and Abelian ideals the type is an affine simple root. We give several descriptions of the set of edges of given type and uniform expressions for the number of edges. By a result of Peterson, the number of Abelian ideals is $2^{n}$, where $n$ is the rank of $\Delta$. We prove that the number of edges of the corresponding Hasse diagram is $(n+1) 2^{n-2}$. For $\Delta^{+}$and the Abelian ideals, we compute the number of edges of each type and prove that the number of edges of type $\alpha$ depends only on the length of the root $\alpha$.
\end{abstract}

Keywords Simple Lie algebra $\cdot$ Root system $\cdot$ Hasse diagram $\cdot$ Ad-nilpotent ideal

Let $\Delta$ be a reduced irreducible root system in an $n$-dimensional real vector space $V$. Choose a subsystem of positive roots $\Delta^{+}$with the corresponding subset of simple roots $\Pi=\left\{\alpha_{1}, \ldots, \alpha_{n}\right\}$. Let $G$ be the corresponding simply-connected simple algebraic group with Lie algebra $\mathfrak{g}$. Fix a triangular decomposition $\mathfrak{g}=\mathfrak{u}^{+} \oplus \mathfrak{t} \oplus \mathfrak{u}^{-}$, where $\mathfrak{t}$ is a Cartan subalgebra and $\mathfrak{u}^{+}=\bigoplus_{\gamma \in \Delta^{+}} \mathfrak{g}_{\gamma}$. Then $\mathfrak{b}=\mathfrak{t} \oplus \mathfrak{u}^{+}$is the fixed Borel subalgebra. The root order in $V$ is given by letting $x \preccurlyeq y$ if $y-x$ is a non-negative integral combination of positive roots. In particular, we always regard $\Delta^{+}$as poset under ' $\preccurlyeq$ '. Then the highest root, $\theta$, is the unique maximal element of $\Delta^{+}$, whereas the simple roots are precisely the minimal elements.

In this article, we consider combinatorial properties of several posets associated with $\Delta^{+}$. We will especially be interested in the edges of the Hasse diagrams of these posets. Given a poset $\mathcal{P}$, we write $\mathcal{H}(\mathcal{P})$ for the Hasse diagram of $\mathcal{P}$ and $\mathcal{E}(\mathcal{P})$ for the set of edges of $\mathcal{H}(\mathcal{P})$.

This research was supported in part by CRDF Grant no. RM1-2543-MO-03

D. I. Panyushev $(\bowtie)$

Independent University of Moscow, Bol'shoi Vlasevskii per. 11,

119002 Moscow, Russia

e-mail: panyush@mccme.ru 
The common property of all posets to be considered below is that one can naturally define the type of any edge of $\mathcal{H}(\mathcal{P})$. If $T$ is the parameter set of possible types, then $\mathcal{E}(\mathcal{P})=\sqcup_{t \in T} \mathcal{E}(\mathcal{P})_{t}$, and our aim is to describe this partition.

In the simplest case, the poset in question is $\Delta^{+}$itself. If $v$ covers $\mu$ in $\Delta^{+}$, then the type of the edge $(v, \mu)$ is the simple root $v-\mu$. Let $h$ (resp. $\left.h^{*}\right)$ denote the Coxeter (resp. dual Coxeter) number of $\Delta$. We show that if $\alpha$ is long, then $\# \mathcal{E}\left(\Delta^{+}\right)_{\alpha}=h^{*}-2$; if $\alpha$ is short, then

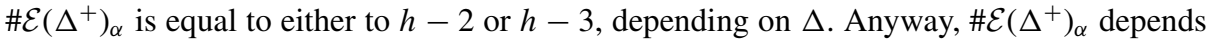
only on $\|\alpha\|$, the length of $\alpha$. Although these results are not difficult, they are apparently new, see Section 1. A similar phenomenon occurs in several related cases. First, if $\Delta^{+}$is not simply-laced, then one can consider the subposet of $\Delta^{+}$consisting of all short roots. More generally, given a simple $G$-module $\mathbb{V}$, one can consider the poset of weights of $\mathbb{V}$, see Section 2.

Another interesting poset is that of all ad-nilpotent ideals in $\mathfrak{b}$, denoted by $\mathfrak{A} \mathfrak{d}$. There has recently been a lot of activity in studying ad-nilpotent ideals [4-6, 9-11, 13, 17, 20]. Combinatorially, $\mathfrak{A d}$ is the set of all upper (=dual order) ideals of $\Delta^{+}$, the partial order being given by the usual containment. An edge of $\mathcal{H}(\mathfrak{A} \mathfrak{d})$ is a pair of ad-nilpotent ideals $\left(\mathfrak{c}, \mathfrak{c}^{\prime}\right)$ such that $\mathfrak{c}^{\prime} \subset \mathfrak{c}$ and $\operatorname{dim} \mathfrak{c}=\operatorname{dim} \mathfrak{c}^{\prime}+1$. We then say that the edge $\left(\mathfrak{c}, \mathfrak{c}^{\prime}\right)$ terminates in $\mathfrak{c}$. It is easily seen that the edges terminating in $\mathfrak{c}$ are in a bijection with the generators of $\mathfrak{c}$, i.e., the minimal roots in the corresponding upper ideal $I_{\mathfrak{c}}$. To define the type of edges in this situation, we exploit the following ingredients:

- a bijection between the ad-nilpotent ideals and certain elements of the affine Weyl group, $\hat{W}$ (Cellini-Papi [4]; in the Abelian case this goes back to Peterson and Kostant [9]). Given $\mathfrak{c} \in \mathfrak{A} \mathfrak{d}$, let $w_{\mathfrak{c}}$ denote the corresponding element of $\hat{W}$.

- the characterisation of the generators of $\mathfrak{c}$ in terms of $w_{\mathfrak{c}}$ [11].

Now, the type of an edge appears to be an affine simple root. The set of affine simple roots is $\hat{\Pi}=\left\{\alpha_{0}\right\} \cup \Pi$, where $\alpha_{0}$ is an extra root, see details in Section 3. We give a closed formula for the number of edges and provide several geometric descriptions for the set of edges of a given type. The first of them is based on a relationship between $\mathfrak{A} \mathfrak{d}$ and the integral points of the simplex $D_{\min }$; second description uses the action of $w_{\mathfrak{c}}$ on the fundamental alcove in $V$, see Theorems 3.8 and 3.9. The last description is given in terms of reduced decompositions of $w_{\mathfrak{c}}$. Namely, $\mathcal{H}(\mathfrak{A} \mathfrak{d})$ has an edge of type $\alpha_{i}$ terminating in $\mathfrak{c}$ if and only if $w_{\mathfrak{c}}$ has a reduced decomposition starting with $s_{i}$. Here $s_{i} \in \hat{W}$ is the reflection corresponding to $\alpha_{i} \in \hat{\Pi}$.

The subposet of $\mathfrak{A} \mathfrak{d}$ consisting of all Abelian ideals is denoted by $\mathfrak{A} \mathfrak{b}$. Because $\mathcal{H}(\mathfrak{A} \mathfrak{b})$ is a subdiagram of $\mathcal{H}(\mathfrak{A} \mathfrak{d})$, the type of edges in $\mathcal{H}(\mathfrak{A} \mathfrak{b})$ is well-defined. By a result of D. Peterson, $\# \mathfrak{A} \mathfrak{b}=2^{n}$ (see $[4,9]$ ). We first show conceptually that the number of edges of $\mathcal{H}(\mathfrak{A} \mathfrak{b})$ equals $(n+1) 2^{n-2}$. Our next result asserts that $\# \mathcal{E}(\mathfrak{A} \mathfrak{b})_{\alpha}, \alpha \in \hat{\Pi}$, depends only on $\|\alpha\|$. Furthermore, if $\mathfrak{g} \neq \mathfrak{s p}_{2 n}$, then the number of edges of each type is $2^{n-2}$. Unfortunately, the proof consists of explicit verifications for all simple Lie algebras. To this end, we choose the following path. A root $\gamma \in \Delta^{+}$is said to be commutative if the upper ideal generated by $\gamma$ is Abelian. The commutative roots form an upper ideal, which is not necessarily Abelian. (We notice that the complement of this ideal has a unique maximal element, which can explicitly be described; a uniform description of the cardinality of the set of commutative roots is also given, see Theorem 4.4.) The utility of commutative roots is revealed via the following property. Suppose $\mathfrak{a} \in \mathfrak{A} \mathfrak{b}$ and $\gamma$ is a generator of $\mathfrak{a}$. By the general rule mentioned above for $\mathfrak{A} \mathfrak{d}$, the pair $(\mathfrak{a}, \gamma)$ determines an edge terminating in $\mathfrak{a}$. If this edge is of type $\alpha$, then we say that $\gamma$ has class $\alpha$ (in the Abelian ideal $\mathfrak{a}$ ). The point here is that the class of $\gamma$ does not depend on an Abelian ideal in which $\gamma$ is a generator. Thus, each commutative root gains a well-defined class, which is an element of $\hat{\Pi}$. Furthermore, we provide effective methods of 
computing the class of commutative roots. Our strategy for computing the numbers $\# \mathcal{E}(\mathfrak{A} \mathfrak{b})_{\alpha}$, $\alpha \in \hat{\Pi}$, begins with determining all commutative roots of class $\alpha$, say $\left\{\gamma_{1}, \ldots, \gamma_{t}\right\}$. Then we compute, for each $i$, the number of Abelian ideals having $\gamma_{i}$ as a generator. The sum of all these numbers equals $\# \mathcal{E}(\mathfrak{A} \mathfrak{b})_{\alpha}$. For the classical Lie algebras the description of classes of the commutative roots is given using the usual matrix realisations; for the exceptional cases, we list the classes in the Appendix.

In Section 5, we introduce the covering polynomial of a finite poset, and compute this polynomial for the posets considered in this article.

\section{Edges of the Hasse diagram of $\Delta^{+}$}

Let $\mathcal{H}=\mathcal{H}\left(\Delta^{+}\right)$be the Hasse diagram of $\left(\Delta^{+}, \preccurlyeq\right)$. In other words, $\mathcal{H}$ is a directed graph whose set of vertices is $\Delta^{+}$, and the set of edges, $\mathcal{E}(\mathcal{H})$, consists of the pairs of positive roots $(\mu, v)$ such that $\mu-v \in \Pi$. We then say that $(\mu, v)$ is an edge of type $\mu-v$.

Let $h=h(\Delta)=h(\mathfrak{g})$ denote the Coxeter number of $\Delta$ (or $\mathfrak{g}$ ). As is well-known, the number of vertices of $\mathcal{H}$ equals $n h / 2$.

Theorem 1.1. Suppose $\Delta$ is simply-laced. Then the number of edges of each type in $\mathcal{H}\left(\Delta^{+}\right)$ is equal to $h-2$. The total number of edges equals $n(h-2)$.

Proof: Clearly, the number of edges of type $\alpha$ is equal to the number of positive roots $v$ such that $v+\alpha \in \Delta^{+}$. Since all roots have the same length, the latter is the same as the number of positive roots $v$ such that $(\nu, \alpha)<0$.

For any $\gamma \in \Delta$, we set $\Delta(\gamma)=\{\mu \in \Delta \mid(\mu, \gamma) \neq 0\}$. By [2, chap. VI, $\S 11$, Prop. 32], we have $\# \Delta(\gamma)=4 h-6$ for any $\gamma$. Therefore the number of such positive roots is $2 h-3$. Consider the partition of this set according to the sign of the scalar product with $\gamma$ :

$$
\Delta(\gamma)^{+}=\Delta(\gamma)_{>0}^{+} \sqcup \Delta(\gamma)_{<0}^{+}
$$

Now, let $\gamma=\alpha$ be a simple root. Then $\alpha \in \Delta(\alpha)_{>0}^{+}$, and it is easily seen that the reflection $s_{\alpha}$ yields a bijection:

$$
s_{\alpha}: \Delta(\alpha)_{>0}^{+} \backslash\{\alpha\} \rightarrow \Delta(\alpha)_{<0}^{+}
$$

Hence $\# \Delta(\alpha)_{<0}^{+}=h-2$. But this is exactly the number of edges of type $\alpha$.

If $\Delta$ is not simply-laced, then one has to distinguish long and short roots. Let $\Pi_{l}$ (resp. $\Pi_{s}$ ) stand for the set of long (resp. short) simple roots. Let $h^{*}=h^{*}(\Delta)$ be the dual Coxeter number of $\Delta$. By definition, $h^{*}=\left(\rho, \theta^{\vee}\right)+1$. If $\Delta$ is simply-laced, then $h^{*}=h$. The values of $h$ and $h^{*}$ in the non-simply-laced cases are given in the following table

\begin{tabular}{lllrr}
\hline & $\mathbf{B}_{n}$ & $\mathbf{C}_{n}$ & $\mathbf{F}_{4}$ & $\mathbf{G}_{2}$ \\
\hline$h$ & $2 n$ & $2 n$ & 12 & 6 \\
$h^{*}$ & $2 n-1$ & $n+1$ & 9 & 4 \\
\hline
\end{tabular}


Theorem 1.2. Suppose $\Delta$ is not simply-laced.

(i) For $\alpha \in \Pi_{l}$, the number of edges of type $\alpha$ in $\mathcal{H}\left(\Delta^{+}\right)$equals $h^{*}-2$.

(ii) For $\alpha \in \Pi_{s}$, the number of edges of type $\alpha$ in $\mathcal{H}\left(\Delta^{+}\right)$equals

$$
\left\{\begin{array}{l}
h-2, \quad \text { if } \Delta \in\left\{\mathbf{B}_{n}, \mathbf{C}_{n}, \mathbf{F}_{4}\right\}, \\
h-3, \quad \text { if } \Delta=\mathbf{G}_{2} .
\end{array}\right.
$$

Proof: (i) If $\alpha \in \Pi_{l}$, then the number of edges of type $\alpha$ is again equal to the number of $v \in \Delta^{+}$such that $(v, \alpha)<0$. Therefore we can argue as in Theorem 1.1 . The only difference is that now we refer to [18] for the equality \# $\Delta(\alpha)=4 h^{*}-6$.

(ii) For $\alpha \in \Pi_{s}$, it is no longer true that the number of edges of type $\alpha$ equals the number of $v \in \Delta^{+}$such that $(v, \alpha)<0$. For, it may happen that $(v, \alpha) \geqslant 0$, but $v+\alpha$ is a root. So we give a case-by-case argument. Our notation and numbering of simple roots follows [21, Tables].

- In case of $\mathbf{B}_{n}$, we have $\alpha_{n}=\varepsilon_{n}$ is the only short simple root. The set of roots $\mu$ such that $\mu+\alpha_{n}$ is a root consists of $\varepsilon_{i}-\varepsilon_{n}(i<n), \varepsilon_{1}, \ldots, \varepsilon_{n-1}$. Thus, there are $2 n-2$ such roots.

- In case of $\mathbf{C}_{n}$, we have $\alpha_{i}=\varepsilon_{i}-\varepsilon_{i+1} \in \Pi_{s}$, $(i<n)$. The set of roots $\mu$ such that $\mu+\alpha_{i}$ is a root consists of $\varepsilon_{k}-\varepsilon_{i}(k<i) ; \varepsilon_{i+1}-\varepsilon_{l}(i+1<l) ; \varepsilon_{i+1}+\varepsilon_{m}$ for all $m$. Thus, there are $2 n-2$ possibilities for each $i$.

The two exceptional cases are left to the reader. (The Hasse diagram for the $\mathbf{F}_{4}$-case is depicted below.)

\section{Remark 1.}

1. In the following section we prove $a$ priori (in a slightly more general context) that the number of edges depends only on the length of a simple root, see Theorem 2.2.

2. It is not hard to find an isomorphism between the posets $\Delta^{+}\left(\mathbf{B}_{n}\right)$ and $\Delta^{+}\left(\mathbf{C}_{n}\right)$. Their Hasse diagrams are therefore isomorphic, too, and hence have the same number of edges. However, this isomorphism does not respect the length of roots and type of edges.

3. The second claim of Theorem 1.2 for $\Delta \in\left\{\mathbf{B}_{n}, \mathbf{C}_{n}, \mathbf{F}_{4}\right\}$ admits a partial explanation. Suppose $\alpha \in \Pi_{s}$ and $e_{\alpha} \in \mathfrak{g}_{\alpha}$ is a nonzero root vector. Then $\left(\operatorname{ad} e_{\alpha}\right)^{3}=0$, and an easy calculation shows that the number of edges of type $\alpha$ is equal to $\left(\operatorname{dim} G \cdot e_{\alpha}-2\right) / 2$. For these three cases, there is an interesting phenomenon observed by R. Brylinski and B. Kostant [3]. The orbit $G \cdot e_{\alpha} \subset \mathfrak{g}$ is shared in the following sense. Let $\tilde{\mathfrak{g}}$ be the simple Lie algebra obtained from (the Dynkin diagram of $\mathfrak{g}$ by unfolding, i.e., $\mathbf{B}_{n} \mapsto \mathbf{D}_{n+1}, \mathbf{C}_{n} \mapsto \mathbf{A}_{2 n-1}$, $\mathbf{F}_{4} \mapsto \mathbf{E}_{6}$. Then the minimal nilpotent $\tilde{G}$-orbit in $\tilde{\mathfrak{g}}$ is a finite covering of $G \cdot e_{\alpha}$. Furthermore, we have $h(\mathfrak{g})=h(\tilde{\mathfrak{g}})$. Finally, since $\tilde{\mathfrak{g}}$ is simply-laced, the dimension of the minimal nilpotent orbit in $\tilde{\mathfrak{g}}$ equals $2 h(\tilde{\mathfrak{g}})-2$.

Example 1.3. The Hasse diagram $\mathcal{H}\left(\Delta^{+}\left(\mathbf{F}_{4}\right)\right)$ has 34 edges, see Figure 1 . The edges of the same type are drawn to have the same slope. Therefore we have indicated only the type of 4 edges in the upper part of the diagram. The black nodes represent long roots. For brevity, the root $\sum_{i=1}^{4} m_{i} \alpha_{i}$ is denoted by $\left[\begin{array}{llll}m_{1} & m_{2} & m_{3} & m_{4}\end{array}\right]$. 


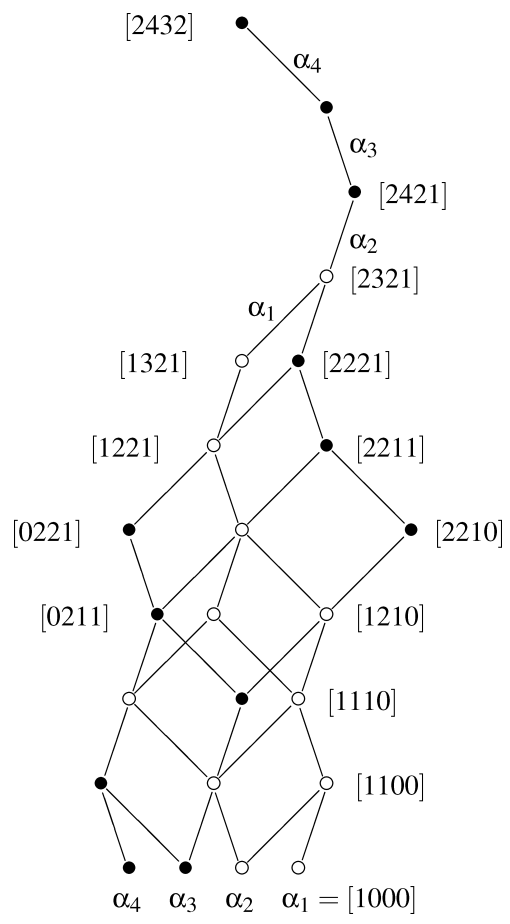

Fig. 1 The Hasse diagram of $\Delta^{+}\left(\mathbf{F}_{4}\right)$

\section{Some generalisations}

Suppose $\Delta^{+}$has roots of different length. We use subscripts 's' and 'l' to mark various objects related to short and long roots, respectively. For instance, $\Delta_{s}$ is the set of short roots, $\Delta=\Delta_{s} \sqcup \Delta_{l}$, and $\Pi_{s}=\Pi \cap \Delta_{s}$. Set $\rho_{s}=\frac{1}{2}\left|\Delta_{s}^{+}\right|$and $\rho_{l}=\frac{1}{2}\left|\Delta_{l}^{+}\right|$. Let $\theta \in \Delta^{+}$be the highest root and $\theta_{s}$ the short dominant root. Recall that $\Delta_{l}=W \cdot \theta, \Delta_{s}=W \cdot \theta_{s}$, and $\|\theta\|^{2} /\left\|\theta_{s}\right\|^{2}=2$ or 3 . Consider $\Delta_{s}^{+}$as subposet of $\Delta^{+}$. This poset has a geometric meaning, see below. Let me stress that although the vertices of $\mathcal{H}\left(\Delta_{s}^{+}\right)$represent the short roots only, the edges still correspond to the whole of $\Pi$. It follows from [2, ch.VI, $\S 1$, Prop. 33] that $\#\left(\Delta_{s}^{+}\right)=h \cdot \#\left(\Pi_{s}\right)$. Let us now compute the number of edges of each type.

\section{Theorem 2.1.}

(i) For $\alpha \in \Pi_{s}$, the number of edges of type $\alpha$ in $\mathcal{H}\left(\Delta_{s}^{+}\right)$is $h\left(\Delta_{s}\right)-2$.

(ii) For $\alpha \in \Pi_{l}$, the number of edges of type $\alpha$ in $\mathcal{H}\left(\Delta_{s}^{+}\right)$is $h^{*}(\Delta)-h\left(\Delta_{l}\right)$.

\section{Proof:}

(i) An edge of type $\alpha \in \Pi_{s}$ is a pair $(\gamma, \mu) \in \Delta_{s}^{+} \times \Delta_{s}^{+}$such that $\gamma-\mu=\alpha$. Hence the number of edges of type $\alpha$ is equal to $\#\left\{\mu \in \Delta_{s}^{+} \mid(\mu, \alpha)<0\right\}$. Recall that $\Delta_{s}$ is a root system in its own right. It can be reducible, but all irreducible subsystems are isomorphic, since $W$ acts transitively on the roots of the same length. Therefore the Coxeter number $h\left(\Delta_{s}\right)$ is well-defined. Furthermore, $\Pi_{s}$ forms a part of a basis for $\Delta_{s}^{+}$. Hence one may refer to Theorem 1.1. 
(ii) If $\alpha \in \Pi_{l}$, then again the number of edges of type $\alpha$ is equal to

$$
\begin{aligned}
\#\left\{\mu \in \Delta_{s}^{+} \mid(\alpha, \mu)<0\right\} & =\#\left\{\mu \in \Delta^{+} \mid(\alpha, \mu)<0\right\}-\#\left\{\mu \in \Delta_{l}^{+} \mid(\alpha, \mu)<0\right\}= \\
& =\left(h^{*}(\Delta)-2\right)-\left(h\left(\Delta_{l}\right)-2\right)=h^{*}(\Delta)-h\left(\Delta_{l}\right) .
\end{aligned}
$$

Here we have also used the fact that $\Delta_{l}$ is a root system and $h\left(\Delta_{l}\right)$ is well-defined.

Remark. There is the obvious recipe for obtaining $\mathcal{H}\left(\Delta_{s}^{+}\right)$from $\mathcal{H}\left(\Delta^{+}\right)$. One has to erase all vertices (with connecting edges) corresponding to the long roots.

It is easy to realise that one can consider similar problems for arbitrary representations of simple Lie algebras. Let $\mathbb{V}_{\lambda}$ be the simple finite-dimensional $\mathfrak{g}$-module with highest weight $\lambda$. We write $m_{\lambda}(\mu)$ for the dimension of $\mu$-weight space of $\mathbb{V}_{\lambda}$. The set of weights of $\mathbb{V}_{\lambda}$ is denoted by $\mathcal{P}\left(\mathbb{V}_{\lambda}\right)$. Being a subset of $\mathcal{V}, \mathcal{P}\left(\mathbb{V}_{\lambda}\right)$ can be regarded as poset under ' $\preccurlyeq$ '. The weight diagram of $\mathbb{V}_{\lambda}$, denoted $\mathcal{W}\left(\mathbb{V}_{\lambda}\right)$, is the directed multigraph whose set of vertices is $\mathcal{P}\left(\mathbb{V}_{\lambda}\right)$ and the set of edges consists of the pairs $(v, \mu)$ such that $v-\mu \in \Pi$. The multiplicity of the edge $(\nu, \mu)$ is defined as $\min \left\{m_{\lambda}(\mu), m_{\lambda}(\nu)\right\}$. It is easy to check that $\mathfrak{W}\left(\mathbb{V}_{\lambda}\right)$ is a simple graph (i.e., all the multiplicities are equal to 1 ) if and only if $m_{\lambda}(\mu)=1$ for all nonzero $\mu \in \mathcal{P}\left(\mathbb{V}_{\lambda}\right)$. If $\mathcal{W}\left(\mathbb{V}_{\lambda}\right)$ is a simple graph, then it is nothing but the Hasse diagram of $\mathcal{P}\left(\mathbb{V}_{\lambda}\right)$. As above, we define the "type" of each edge of $\mathfrak{W}\left(\mathbb{V}_{\lambda}\right)$.

Theorem 2.2. If $\alpha, \beta \in \Pi$ have the same length, then the number of edges of type $\alpha$ and $\beta$ in $\mathcal{W}\left(\mathbb{V}_{\lambda}\right)$ (counted with multiplicities) is the same. That is to say, the number of edges of type $\alpha \in \Pi$ depends only on the length of $\alpha$.

Proof: Let $\mathfrak{s l}_{2}(\alpha)$ be the simple three-dimensional subalgebra of $\mathfrak{g}$ corresponding to $\alpha$. Consider $\mathbb{V}_{\lambda}$ as $\mathfrak{s l}_{2}(\alpha)$-module. We write $R(d)$ for the simple $\mathfrak{s l}_{2}$-module of dimension $d+1$. It is easy to check that if $\left.\mathbb{V}_{\lambda}\right|_{\mathfrak{s l}_{2}(\alpha)} \simeq \oplus n_{i} R\left(d_{i}\right)$, then the number of edges of type $\alpha$ is equal to $\sum_{i} n_{i} d_{i}$. On the other hand, the subalgebras $\mathfrak{s l}_{2}(\alpha)$ and $\mathfrak{s l}_{2}(\beta)$ are conjugate under Aut $\mathfrak{g}$, so that $\left.\mathbb{V}_{\lambda}\right|_{\mathfrak{s l}_{2}(\alpha)}$ and $\left.\mathbb{V}_{\lambda}\right|_{\mathfrak{s l}_{2}(\mu)}$ are isomorphic as $\mathfrak{s l}_{2}$-modules.

\section{Example 2.3.}

1. If $\mathbb{V}_{\lambda}=\mathfrak{g}$ is the adjoint $\mathfrak{g}$-module, then $\mathcal{P}(\mathfrak{g})=\Delta \cup\{0\}$. Here $\mathfrak{W}(\mathfrak{g})$ is a simple graph. After deleting $\{0\}$ from this weight diagram, we obtain two isomorphic connected components corresponding to $\Delta^{+}$and $\Delta^{-}$. This provides another (a priori) proof for the fact that the number of edges in $\mathcal{H}\left(\Delta^{+}\right)$of type $\alpha \in \Pi$ depends only on the length of $\alpha$. Clearly, we loose two edges of each type with deleting the vertex $\{0\}$. Therefore it follows from Theorem 1.2 that the number of edges in $\mathfrak{W}(\mathfrak{g})$ of type $\alpha \in \Pi_{l}$ is equal to $2\left(h^{*}(\Delta)-2\right)+2=2 h^{*}(\Delta)-2$.

2. For $\mathbb{V}_{\theta_{s}}$, we have $\mathcal{P}\left(\mathbb{V}_{\theta_{s}}\right)=\Delta_{s} \cup\{0\}$. Here again $\mathfrak{W}\left(\mathbb{V}_{\theta_{s}}\right)$ is a simple graph. After deleting $\{0\}$ from this weight diagram, we obtain two isomorphic connected components corresponding to $\Delta_{s}^{+}$and $\Delta_{s}^{-}$, etc. The difference with the previous example is that we loose only two edges of each tshort type after removing $\{0\}$.

3. Let $\mathfrak{g}$ be of type $\mathbf{B}_{n}$ and $\lambda=\varphi_{n}$. Here the $\mathfrak{g}$-module $\mathbb{V}_{\varphi_{n}}$ is weight multiplicity free, $\operatorname{dim} \mathbb{V}_{\varphi_{n}}=2^{n}$, and the weights are $\frac{1}{2}\left( \pm \varepsilon_{1} \pm \varepsilon_{2} \ldots \pm \varepsilon_{n}\right)$. The simple root $\alpha_{n}=\varepsilon_{n}$ can be subtracted from a weight of this representation if and only if the last coordinate has sign 
+ . Hence the number of edges of type $\alpha_{n}$ in $\mathfrak{W}\left(\mathbb{V}_{\varphi_{n}}\right)$ equals $2^{n-1}$. Similarly, one computes that the number of edges of any other type equals $2^{n-2}$.

4. Let $\mathfrak{g}$ be of type $\mathbf{E}_{6}$ and $\lambda=\varphi_{1}$. Here $\operatorname{dim} \mathbb{V}_{\varphi_{1}}=27$. Then $\mathcal{P}\left(\mathbb{V}_{\varphi_{1}}\right)$ is a so-called minuscule poset and $\mathfrak{W}\left(\mathbb{V}_{\varphi_{1}}\right)$ has 36 edges. Surely, we have 6 edges of each type.

\section{The poset of ad-nilpotent ideals of $\mathfrak{b}$}

There is another natural poset attached to $\Delta^{+}$, where one can define the type of edges in the Hasse diagram. As we shall see, this leads to interesting combinatorial results.

Recall that $\Delta^{+}$is equipped with the partial order " $\preccurlyeq$ ". A subset $S \subset \Delta^{+}$is called an upper ideal, if the conditions $\gamma \in S$ and $\gamma \preccurlyeq \tilde{\gamma}$ imply $\tilde{\gamma} \in S$. The geometric counterpart of an upper ideal is an ad-nilpotent ideal. A subspace $\mathfrak{c} \subset \mathfrak{b}$ is said to be an ad-nilpotent ideal (of $\mathfrak{b}$ ), if it is contained in $\mathfrak{u}^{+}$and satisfies the condition $[\mathfrak{b}, \mathfrak{c}] \subset \mathfrak{c}$. If $\mathfrak{c}$ is an ad-nilpotent ideal, then $\mathfrak{c}=\bigoplus_{\gamma \in I_{\mathfrak{c}}} \mathfrak{g}_{\gamma}$, where $I_{\mathfrak{c}}$ is a subset of $\Delta^{+}$. We also say that $I_{\mathfrak{c}}$ is the set of roots of $\mathfrak{c}$. Obviously, one obtains in this way a bijection between the ad-nilpotent ideals of $\mathfrak{b}$ and the upper ideals of $\Delta^{+}$.

The set of all ad-nilpotent ideals is denoted by $\mathfrak{A} \mathfrak{d}$. In view of the above bijection, we may (and will) identify $\mathfrak{A} \mathfrak{d}$ with the set of all upper ideals of $\Delta^{+}$. Whenever we wish to explicitly indicate that $\mathfrak{A} \mathfrak{d}$ depends on $\mathfrak{g}$, we write $\mathfrak{A} \mathfrak{d}(\mathfrak{g})$. We regard $\mathfrak{A} \mathfrak{d}$ as poset under the usual containment. For instance, $\mathfrak{u}^{+}$(or $\Delta^{+}$) is the unique maximal element of $\mathfrak{A} \mathfrak{d}$. It was shown in [5] that

$$
\# \mathfrak{A} \mathfrak{d}=\prod_{i=1}^{n} \frac{h+e_{i}+1}{e_{i}+1},
$$

where $e_{1}, \ldots, e_{n}$ are the exponents of $\Delta^{+}$. Let $\mathcal{E}(\mathfrak{A} \mathfrak{d})$ denote the set of edges of the Hasse diagram $\mathcal{H}(\mathfrak{A} \mathfrak{d})$. Clearly, a pair of ad-nilpotent ideals $\mathfrak{c}, \mathfrak{c}^{\prime}$ gives rise to an edge of $\mathcal{H}(\mathfrak{A} \mathfrak{d})$ if and only if $\mathfrak{c}^{\prime} \subset \mathfrak{c}$ and $\operatorname{dim} \mathfrak{c}=\operatorname{dim} \mathfrak{c}^{\prime}+1$. Combinatorially: $I_{\mathfrak{c}^{\prime}} \subset I_{\mathfrak{c}}$ and $\# I_{\mathfrak{c}}=\# I_{\mathfrak{c}^{\prime}}+1$. Hence $I_{\mathfrak{c}}=I_{\mathfrak{c}^{\prime}} \cup\{\gamma\}$. It is easily seen that $I_{\mathfrak{c}} \backslash\{\gamma\}$ is again an upper ideal if and only if $\gamma$ is a generator of $\mathfrak{c}$ in the sense of the following definition.

An element $\gamma \in I_{\mathfrak{c}}$ is called a generator of $\mathfrak{c}$ (or $I_{\mathfrak{c}}$ ), if $\gamma-\alpha \notin I$ for any $\alpha \in \Pi$. In other words, $\gamma$ is a minimal element of $I_{\mathfrak{c}}$ with respect to “ß”. We write $\Gamma(\mathfrak{c})$ for the set of generators of $\mathfrak{c}$. Hence $\mathfrak{e}=\left(\mathfrak{c}, \mathfrak{c}^{\prime}\right)$ is an edge of $\mathcal{H}(\mathfrak{A} \mathfrak{d})$ if and only if $I_{\mathfrak{c}^{\prime}}=I_{\mathfrak{c}} \backslash\{\gamma\}$ for some $\gamma \in \Gamma(\mathfrak{c})$. In this case we also say that $\mathfrak{e}$ originates in $\mathfrak{c}^{\prime}$ and terminates in $\mathfrak{c}($ or, $\mathfrak{c}$ is the terminating ideal of $\mathfrak{e})$. Thus, we have proved

Proposition 3.2. There is a bijection between $\mathcal{E}(\mathfrak{A} \mathfrak{d})$ and the set of pairs $(\mathfrak{c}, \gamma)$, where $\mathfrak{c} \in \mathrm{Ad}$ and $\gamma \in \Gamma(\mathfrak{c})$. More precisely, for any $\mathfrak{c} \in \mathfrak{A} \mathfrak{d}$, the edges terminating in $\mathfrak{c}$ are in a bijection with $\Gamma(\mathfrak{c})$.

In [11, Section 6], we introduced, for each simple Lie algebra $\mathfrak{g}$, a generalised Narayana polynomial $\mathcal{N}_{\mathfrak{g}}$. By definition, it is the generating function that counts the ad-nilpotent ideals with respect to the number of generators, i.e.,

$$
\mathcal{N}_{\mathfrak{g}}(q)=\sum_{i=0}^{n} \#\{\mathfrak{c} \in \mathfrak{A} \mathfrak{d}(\mathfrak{g}) \mid \# \Gamma(\mathfrak{c})=k\} \cdot q^{k}
$$


In case $\mathfrak{g}=\mathfrak{s l}_{n+1}$, one obtains the Narayana polynomials ( $q$-analogues of the Catalan number). Obviously, $\mathcal{N}_{\mathfrak{g}}(1)=\# \mathfrak{A} \mathfrak{d}(\mathfrak{g})$. The following readily follows from the definition of $\mathcal{N}_{\mathfrak{g}}$ and Proposition 3.2.

Proposition 3.3. $\left.\frac{d}{d q} \mathcal{N}_{\mathfrak{g}}(q)\right|_{q=1}=\# \mathcal{E}(\mathfrak{A} \mathfrak{d}(\mathfrak{g}))$.

\section{Corollary 3.4.}

$$
\# \mathcal{E}(\mathfrak{A} \mathfrak{d}(\mathfrak{g}))=\frac{n}{2} \# \mathfrak{A} \mathfrak{d}(\mathfrak{g})=\frac{n}{2} \prod_{i=1}^{n} \frac{h+e_{i}+1}{e_{i}+1}
$$

Proof: It was observeed in [11, Section 6] that $\mathcal{N}_{\mathfrak{g}}(q)=\sum_{i=0}^{n} a_{i} q^{i}$ is a palindromic polynomial, i.e., $a_{j}=a_{n-j}$ for all $j$. But it is easily seen that $f^{\prime}(1)=\frac{\operatorname{deg} f}{2} f(1)$ for any palindromic polynomial $f$.

Below, we provide the numbers $\# \mathfrak{A} \mathfrak{d}(\mathfrak{g})$ and $\# \mathcal{E}(\mathfrak{A} \mathfrak{d}(\mathfrak{g}))$ for all simple Lie algebras.

\begin{tabular}{lcccccccc}
\hline & $\mathbf{A}_{n}$ & $\mathbf{B}_{n}, \mathbf{C}_{n}$ & $\mathbf{D}_{n}$ & $\mathbf{E}_{6}$ & $\mathbf{E}_{7}$ & $\mathbf{E}_{8}$ & $\mathbf{F}_{4}$ & $\mathbf{G}_{2}$ \\
\hline$\# \mathfrak{A} \mathfrak{d}$ & $\frac{1}{n+2}\left(\begin{array}{c}2 n+2 \\
n+1\end{array}\right)$ & $\left(\begin{array}{c}2 n \\
n\end{array}\right)$ & $\left(\begin{array}{c}2 n \\
n\end{array}\right)-\left(\begin{array}{c}2 n-2 \\
n-1\end{array}\right)$ & 833 & 4160 & 25080 & 105 & 8 \\
$\# \mathcal{E}(\mathfrak{A} \mathfrak{d})$ & $\left(\begin{array}{c}2 n+1 \\
n+2\end{array}\right)$ & $n\left(\begin{array}{c}2 n-1 \\
n\end{array}\right)$ & $n\left(\left(\begin{array}{c}2 n-1 \\
n\end{array}\right)-\left(\begin{array}{c}2 n-3 \\
n-1\end{array}\right)\right)$ & 2499 & 14560 & 100320 & 210 & 8 \\
\hline
\end{tabular}

Remark 3.5. In [7], a simple convex polytope $\mathcal{M}(\mathfrak{g})$ is associated to an arbitrary irreducible finite root system or simple Lie algebra (for types $\mathbf{A}_{n}$ and $\mathbf{B}_{n}$ these polytopes were known before). It is curious that the number of vertices of $\mathcal{M}(\mathfrak{g})$ is $\# \mathfrak{A} \mathfrak{d}(\mathfrak{g})$, and the number of edges of $\mathcal{M}(\mathfrak{g})$ is $\# \mathcal{E}(\mathfrak{A} \mathfrak{d}(\mathfrak{g}))$. We do not know of whether there is a more deep connection between $\mathcal{M}(\mathfrak{g})$ and the Hasse diagram $\mathcal{H}(\mathfrak{A} \mathfrak{d}(\mathfrak{g}))$. At least, $\mathcal{H}(\mathfrak{A} \mathfrak{d}(\mathfrak{g}))$ is not isomorphic to the graph of $\mathcal{M}(\mathfrak{g})$.

In order to define the type of an edge, we need some results on a relationship between $\mathfrak{A} \mathfrak{d}$ and certain elements of the affine Weyl group. Let us recall the necessary setup.

We have $V:=\mathfrak{t}_{\mathcal{R}}=\oplus_{i=1}^{n} \mathcal{R} \alpha_{i}$ and ( , ) a $W$-invariant inner product on $V$. As usual, $\mu^{\vee}=2 \mu /(\mu, \mu)$ is the coroot for $\mu \in \Delta$. Then $Q^{\vee}=\oplus_{i=1}^{n} \mathcal{Z} \alpha_{i}^{\vee}$ is the coroot lattice in $V$.

Letting $\hat{V}=V \oplus \mathcal{R} \delta \oplus \mathcal{R} \lambda$, we extend the inner product ( , ) on $\hat{V}$ so that $(\delta, V)=$ $(\lambda, V)=(\delta, \delta)=(\lambda, \lambda)=0$ and $(\delta, \lambda)=1$. Then

$\hat{\Delta}=\{\Delta+k \delta \mid k \in \mathcal{Z}\}$ is the set of affine (real) roots;

$\hat{\Delta}^{+}=\Delta^{+} \cup\{\Delta+k \delta \mid k \geqslant 1\}$ is the set of positive affine roots;

$\hat{\Pi}=\Pi \cup\left\{\alpha_{0}\right\}$ is the corresponding set of affine simple roots.

Here $\alpha_{0}=\delta-\theta$. For $\alpha_{i}(0 \leqslant i \leqslant n)$, let $s_{i}$ denote the corresponding reflection in $G L(\hat{V})$. That is, $s_{i}(x)=x-2\left(x, \alpha_{i}\right) \alpha_{i}^{\vee}$ for any $x \in \hat{V}$. The affine Weyl group, $\hat{W}$, is the subgroup of $G L(\hat{V})$ generated by the reflections $s_{i}, i=0,1, \ldots, n$. If the index of $\alpha \in \hat{\Pi}$ is not specified, then we merely write $s_{\alpha}$. The inner product (, ) on $\hat{V}$ is $\hat{W}$-invariant. The notation $\beta>0$ (resp. $\beta<0$ ) is a shorthand for $\beta \in \hat{\Delta}^{+}$(resp. $\beta \in-\hat{\Delta}^{+}$). The length function on $\hat{W}$ with respect to $s_{0}, s_{1}, \ldots, s_{p}$ is denoted by $\ell$. 
It was proved by Cellini and Papi that there is a bijection between ad-nilpotent ideals and certain elements of $\hat{W}$, see [4]. This can be described as follows.

Given $\mathfrak{c} \in \mathfrak{A} \mathfrak{d}$ with the corresponding upper ideal $I_{\mathfrak{c}} \subset \Delta^{+}$, there is a unique element $w_{\mathfrak{c}} \in \hat{W}$ satisfying the following properties:

$(\diamond)$ For $\gamma \in \Delta^{+}$, we have $\gamma \in I_{\mathfrak{c}}$ if and only if $w_{\mathfrak{c}}(\delta-\gamma)<0$;

(dom) $w_{\mathfrak{c}}(\alpha)>0$ for all $\alpha \in \Pi$;

(min) if $w_{\mathfrak{c}}^{-1}\left(\alpha_{i}\right)=k_{i} \delta+\mu_{i}\left(\alpha_{i} \in \hat{\Pi}\right)$, where $\mu_{i} \in \Delta$ and $k_{i} \in \mathbb{Z}$, then $k_{i} \geqslant-1$.

Following Sommers [17], the element $w_{\mathfrak{c}}$ is said to be the minimal element of $\mathfrak{c}$. The minimal element of $\mathfrak{c}$ can also be characterised as the unique element of $\hat{W}$ satisfying properties $(\diamond)$, (dom), and having the minimal possible length. This explains the term. The elements of $\hat{W}$ satisfying the last two properties are called minimal. The set of minimal elements of $\hat{W}$ is denoted by $\hat{W}_{\min }$. One of the main results of [4] is that the correspondence $\mathfrak{c} \mapsto w_{\mathfrak{c}}$ sets up a bijection between $\mathfrak{A d}$ and $\hat{W}_{\text {min }}$. Conversely, if $w \in \hat{W}_{\text {min }}$, then $\mathfrak{c}_{w}$ stands for the corresponding ad-nilpotent ideal and $I_{w}$ is the set of roots of $\mathfrak{c}_{w}$.

In [11, Theorem 2.2], a characterisation of the generators of $\mathfrak{c}$ was given in terms of $w_{\mathfrak{c}}$. Namely, $\gamma \in \Gamma(\mathfrak{c})$ if and only if $w_{\mathfrak{c}}(\delta-\gamma) \in-\hat{\Pi}$.

Now, we are ready to define the type of an edge in $\mathbb{H}(\mathfrak{A} \mathfrak{d})$.

Definition 3.6. If $\mathfrak{e}=\left(\mathfrak{c}, \mathfrak{c}^{\prime}\right)$ is an edge of $\mathbb{H}(\mathfrak{A} \mathfrak{d})$, with $I_{\mathfrak{c}^{\prime}}=I_{\mathfrak{c}} \backslash\{\gamma\}$, then the type of $\mathfrak{e}$ is the affine simple root $w_{\mathfrak{c}}(\gamma-\delta)$.

Thus, the parameter set for edge types is $\hat{\Pi}$. Let $\mathcal{E}(\mathfrak{A} \mathfrak{d})_{i}$ denote the set of edges of type $\alpha_{i}$, $i=0,1, \ldots, n$. It is a natural problem to find the number of edges of each type. We provide two geometric descriptions of $\mathcal{E}(\mathfrak{A} \mathfrak{d})_{i}$. To this end, we recall another bijection due to Cellini and Papi. Set $D_{\min }=\{x \in V \mid(x, \alpha) \geqslant-1 \forall \alpha \in \Pi \&(x, \theta) \leqslant 2\}$. It is a simplex in $V$.

Proposition 3.7 ( [5, Proposition $2 \& 3])$. There is a natural bijection between $\mathfrak{A d}$ and $D_{\min } \cap Q^{\vee}$.

In [5], this bijection was established using the isomorphism $\hat{W} \simeq W \ltimes Q^{\vee}$ and the affinelinear action of $\hat{W}$ on $V$. This can also be explained entirely in terms of the linear action of $\hat{W}$ on $\hat{V}$. If $w_{\mathfrak{c}}^{-1}\left(\alpha_{i}\right)=\mu_{i}+k_{i} \delta, \quad i=0,1, \ldots, n$, then we define the point $z_{\mathfrak{c}} \in V$ by the equalities $\left(\alpha_{i}, z_{\mathfrak{c}}\right)=k_{i}, i=1, \ldots, n$. (Since $\alpha_{0}=\delta-\theta$ and $\delta$ is $\hat{W}$-invariant, this implies $\left(\theta, z_{\mathfrak{c}}\right)=1-k_{0}$.) Since $w_{\mathfrak{c}} \in \hat{W}_{\min }$, we have $k_{i} \geqslant-1$, hence $z_{\mathfrak{c}} \in D_{\min }$. The correspondence $\mathfrak{c} \mapsto z_{\mathfrak{c}}$ is the required bijection.

Set $F_{i}=\left\{x \in D_{\min } \mid\left(x, \alpha_{i}\right)=-1\right\}, i \geqslant 1$, and $F_{0}=\left\{x \in D_{\min } \mid(x, \theta)=2\right\}$. These are all the facets of $D_{\min }$.

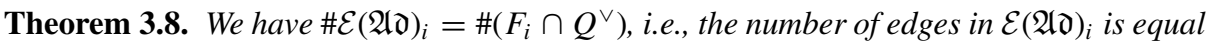
to the number of points $z_{\mathfrak{c}}$ lying in $F_{i}(i=0,1, \ldots, n)$.

Proof: This is a simple combination of preceding results. Clearly, in place of edges of type $\alpha_{i}$ one can describe their terminating ideals. Let $\mathfrak{e}=\left(\mathfrak{c}, \mathfrak{c}^{\prime}\right)$ be an edge of type $\alpha_{i}$, where $I_{\mathfrak{c}^{\prime}}=I_{\mathfrak{c}} \backslash\{\gamma\}$. Then $w_{\mathfrak{c}}(\delta-\gamma)=-\alpha_{i}$. Hence $w_{\mathfrak{c}}^{-1}\left(\alpha_{i}\right)=\gamma-\delta$. Comparing with the above definition of $z_{\mathfrak{c}}$ shows that $z_{\mathfrak{c}} \in F_{i}$. Conversely, if $z_{\mathfrak{c}}$ lies in $F_{i}$, then $w_{\mathfrak{c}}(\delta-\gamma)=\alpha_{i}$ for some $\gamma \in I_{\mathfrak{c}}$. By [11, Theorem 2.2], such $\gamma$ is necessarily a generator of $\mathfrak{c}$, and $\left(I_{\mathfrak{c}}, I_{\mathfrak{c}} \backslash\{\gamma\}\right)$ gives rise to an edge of type $\alpha_{i}$. 
Thus, we have proved that there is an edge of type $\alpha_{i}$ terminating in $\mathfrak{c}$ if and only if $z_{\mathfrak{c}} \in F_{i}$.

Yet another characterisation of the type of an edge can be given using alcoves. Recall that the (open) dominant Weyl chamber is $\mathbb{C}=\{x \in V \mid(x, \alpha)>0 \forall \alpha \in \Pi\}$ and the fundamental alcove is $\mathbb{A}=\{x \in V \mid(x, \alpha)>0 \forall \alpha \in \Pi \&(x, \theta)<1\}$.

For $\gamma \in \Delta^{+}$and $k \in \mathbb{Z}$, we set $\mathbb{H}_{\gamma, k}=\{x \in V \mid(\gamma, x)=k\}$. It is an affine hyperplane in $V$. The connected components of $V \backslash \cup_{\gamma, k} \mathbb{H}_{\gamma, k}$ are called alcoves. As is well-known, $\mathbb{A}$ is one of them, and all alcoves are congruent to $\mathbb{A}$, see e.g. [8]. The walls of $\mathbb{A}$ are $\mathbb{H}_{\alpha_{i}, 0}, \alpha_{i} \in \Pi$, and $\mathbb{H}_{\theta, 1}$. Each wall of $\mathbb{A}$ is equipped with the "type", which is an element of $\hat{\Pi}$. Namely, $\mathbb{H}_{\theta, 1}$ is the wall of type $\alpha_{0}$ and $\mathbb{H}_{\alpha, 0}(\alpha \in \Pi)$ is the wall of type $\alpha$.

Given $\mathfrak{c} \in \mathfrak{A} \mathfrak{d}$, we wish to determine the types of edges terminating in $\mathfrak{c}$. To this end, consider the alcove $w_{\mathfrak{c}}^{-1} * \mathbb{A}$. Here ' $*$ ' stands for the affine-linear action of $\hat{W}$ on $V$. Since $w_{\mathfrak{c}}$ satisfies condition (dom), we have $w_{\mathfrak{c}}^{-1} * \mathbb{A} \subset \mathbb{C}$, see [4].

Theorem 3.9. In the above setting, there is a bijection between the edges of $\mathcal{E}(\mathfrak{A d})$ terminating in $\mathfrak{c}$ and the walls of $w_{\mathfrak{c}}^{-1} * \mathbb{A}$ separating this alcove from the origin. If $H$ is such $a$ wall of $w_{\mathfrak{c}}^{-1} * \mathbb{A}$, then the type of the corresponding edge coincides with the type of the wall $w_{\mathfrak{c}} * H$ of $\mathbb{A}$.

Proof: First, suppose $H=\mathbb{H}_{\gamma, k}$ separates $w_{\mathfrak{c}}^{-1} * \mathbb{A}$ from the origin. Then $w_{\mathfrak{c}}(k \delta-\gamma)<0[4$, Eq. (1.1)]. Let us show that $H$ gives rise to an edge terminating in $\mathfrak{c}$. If $w_{\mathfrak{c}} * \mathbb{H}_{\gamma, k}=\mathbb{H}_{\alpha_{i}, 0}$, then using [14, Eq. (3.3)] we obtain $w_{\mathfrak{c}}(k \delta-\gamma)=-\alpha_{i}$. Since $w_{\mathfrak{c}}$ is minimal, the property (min) forces $k=1$. Hence $\gamma \in \Gamma(\mathfrak{c})$ and the respective edge is of type $\alpha_{i}$. If $w_{\mathfrak{c}} * \mathbb{H}_{\gamma, k}=\mathbb{H}_{\theta, 1}$, then we obtain in a similar way that $k=1$ and $w_{\mathfrak{c}}(k \delta-\gamma)=-\alpha_{0}$. Hence $H$ gives rise to an edge of type $\alpha_{0}$.

Conversely, suppose $\gamma \in \Gamma(\mathfrak{c})$ corresponds to an edge of type $\alpha \in \hat{\Pi}$. Then arguing backwards, we show that $H=\mathbb{H}_{\gamma, 1}$ separates $w_{\mathfrak{c}}^{-1} * \mathbb{A}$ from the origin and $w_{\mathfrak{c}} * H$ is the wall of $\mathbb{A}$ of type $\alpha$.

Finally, we show how to determine the edges terminating in $\mathfrak{c}$ in terms of reduced decompositions of $w_{\mathfrak{c}}$. Suppose that $\ell\left(w_{\mathfrak{c}}\right)=m$, and $w_{\mathfrak{c}}=s_{i_{1}} \ldots s_{i_{m}}$ is a reduced decomposition. It follows from property (dom) that $\alpha_{0}$ is the only simple root that is made negative by $w_{\mathfrak{c}}$. Hence $s_{i_{m}}=s_{0}$. But at the other side we may have several possibilities.

Proposition 3.10. Given $\mathfrak{c}$, there is an edge of type $\alpha_{i}$ terminating in $\mathfrak{c}$ if and only if there is a reduced decomposition of $w_{\mathfrak{c}}$ starting with $s_{i}$.

Proof: Indeed, if $w_{\mathfrak{c}}=s_{i} w^{\prime}$ and $\ell\left(w^{\prime}\right)=\ell\left(w_{\mathfrak{c}}\right)-1$, then $w_{\mathfrak{c}}^{-1}\left(\alpha_{i}\right)<0$. Using properties (min) and (dom), we see that this implies that $w_{\mathfrak{c}}^{-1}\left(\alpha_{i}\right)=\gamma-\delta$ for some $\gamma \in \Delta^{+}$. Hence $\gamma \in \Gamma(\mathfrak{c})$ and the corresponding edge is of type $\alpha_{i}$. This argument can be reversed.

It is not clear as yet how to uniformly compute the number of edges of each type. At least, these numbers can be different for simple roots of the same length.

Example 3.11. For $\mathfrak{g}=\mathfrak{s l}_{4}$, the Hasse diagram of $\mathfrak{A} \mathfrak{d}$ has 21 edges. Their distribution with respect to type is $(5,6,4,6)$, see Figure 2 . The asterisks point out the generators of ideals. 


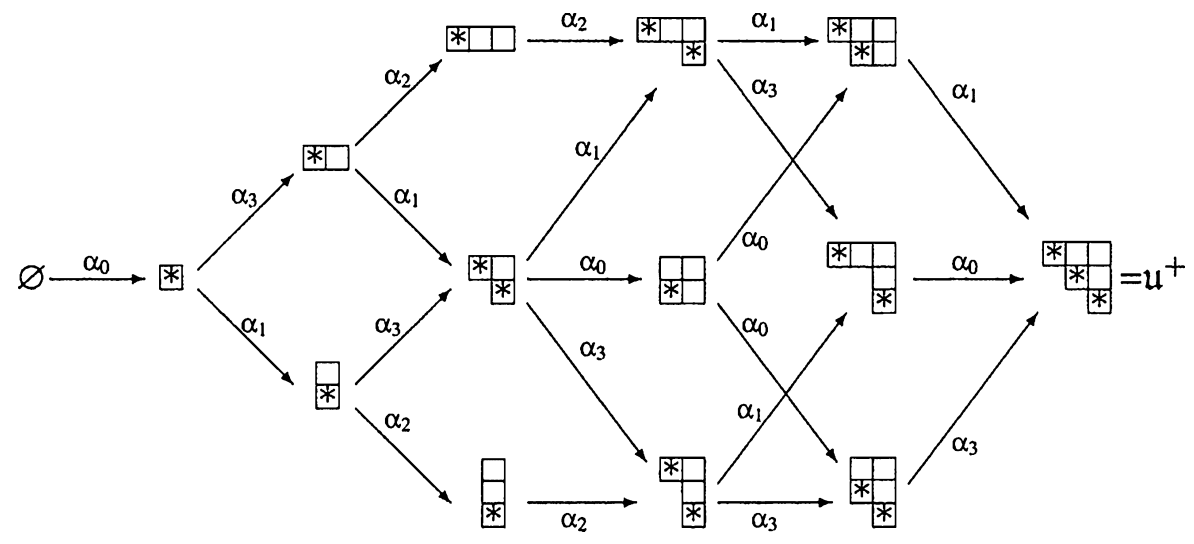

Fig. 2 The Hasse diagram of $\mathfrak{A} \mathfrak{d}\left(\mathfrak{s l}_{4}\right)$ with types of the edges

To compute the type of edges, we use explicit expressions for the minimal elements of all ad-nilpotent ideals. For instance, if $\mathfrak{c}=\mathfrak{u}^{+}$, then the corresponding minimal element is $s_{0} s_{1} s_{3} s_{0} s_{3} s_{1} s_{2} s_{1} s_{3} s_{0}$. It is not hard to find reduced expressions of it starting with $s_{1}$ or $s_{3}$.

\section{The poset of Abelian ideals of $\mathfrak{b}$}

A subspace $\mathfrak{c} \subset \mathfrak{b}$ is called an Abelian ideal, if $[\mathfrak{b}, \mathfrak{c}] \subset \mathfrak{c}$ and $[\mathfrak{c}, \mathfrak{c}]=0$. (It is easily seen that any Abelian ideal is ad-nilpotent, i.e., it is contained in $\mathfrak{u}^{+}$.) In terms of roots, $\mathfrak{c}$ is Abelian if and only if $\left(I_{\mathfrak{c}}+I_{\mathfrak{c}}\right) \cap \Delta^{+}=\varnothing$. The respective upper ideal $I_{\mathfrak{c}}$ is said to be Abelian, too. The sub-poset of $\mathfrak{A} \mathfrak{d}$ consisting of all Abelian ideals is denoted by $\mathfrak{A} \mathfrak{b}$ or $\mathfrak{A} \mathfrak{b}(\mathfrak{g})$. Below, the symbol $\mathfrak{a}$ is used to denote an Abelian ideal.

We keep the notation of the previous section. In particular, to any $\mathfrak{a} \in \mathfrak{A} \mathfrak{b}$ we attach the set of roots $I_{\mathfrak{a}}$, the minimal element $w_{\mathfrak{a}}$, and the alcove $w_{\mathfrak{a}}^{-1} * \mathbb{A} \subset \mathbb{C}$. Following Peterson, an element $w \in \hat{W}_{\min }$ is said to be minuscule, if the corresponding ad-nilpotent ideal is Abelian. Write $\hat{W}_{m c}$ for the set of all minuscule elements. We are going to consider the edges of the Hasse diagram $\mathbb{H}(\mathfrak{A} \mathfrak{b})$ and their types. In this case, our understanding of the situation is better than that in Section 3.

A nice result of D. Peterson asserts that $\# \mathfrak{A} \mathfrak{b}(\mathfrak{g})=2^{\text {rk }} \mathfrak{g}$ for any simple Lie algebra $\mathfrak{g}$. (See [9] and [4] for various proofs). The proof of Cellini and Papi is based on the observation that $\mathfrak{c} \in \mathfrak{A} \mathfrak{d}$ is Abelian if and only if $w_{\mathfrak{c}}^{-1} * \mathbb{A} \subset 2 \mathbb{A}$. It turns out that the number of edges depends only on the rank, too.

Theorem 4.1. If $\mathrm{rk} \mathfrak{g}=n$, then $\# \mathcal{E}(\mathfrak{A} \mathfrak{b}(\mathfrak{g}))=(n+1) 2^{n-2}$.

Proof: Recall that the hyperplanes $\mathbb{H}_{\gamma, k}$ with $\gamma \in \Delta^{+}$and $k \in \mathcal{Z}$ cut $V$ into (open) alcoves congruent to $\mathbb{A}$. In particular, the 'big' simplex $2 \mathbb{A}$ contains $2^{n}$ alcoves. By [4], the alcoves in $2 \mathbb{A}$ bijectively correspond to the Abelian ideals, via the mapping $(\mathfrak{a} \in \mathfrak{A} \mathfrak{b}) \mapsto\left(w_{\mathfrak{a}}^{-1} * \mathbb{A} \subset\right.$ $2 \mathbb{A}$ ). Hence the number of Abelian ideals is $2^{n}$. Consider two alcoves inside $2 \mathbb{A}$ that have a common wall. By Theorem 3.9, this wall gives rise to an edge of $\mathbb{H}(\mathfrak{A} \mathfrak{b})$. Hence the number of edges is equal to the total number of internal walls between alcoves inside $2 \mathbb{A}$. It is an easy exercise to compute this number. (The total number of walls of all these alcoves is $(n+1) 2^{n}$; 
the number of alcove walls on the boundary of $2 \mathbb{A}$ is $(n+1) 2^{n-1}$; all the remaining walls are counted twice. Hence the answer.)

The hyperplanes $\mathbb{H}_{\gamma, k}$ cut $2 \mathbb{A}$ into $2^{n}$ alcoves. It is natural to ask which hyperplanes do meet $2 \mathbb{A}$ ? A partial answer given in [4] says that if $2 \mathbb{A} \cap \mathbb{H}_{\gamma, k} \neq \varnothing$, then $k=1$. Therefore, it remains to characterise the possible roots $\gamma$. This leads to the following definition.

Definition 4.2. Let $\gamma \in \Delta^{+}$. We say that $\gamma$ is commutative if the upper ideal generated by $\gamma$ is Abelian. This Abelian ideal is denoted by $\mathfrak{a}(\gamma)$.

It is easily seen that $\gamma$ is commutative if and and only if there is an Abelian ideal $\mathfrak{a}$ such that $\gamma \in \Gamma(\mathfrak{a})$. Clearly, the set of all commutative roots forms an ad-nilpotent ideal. This adnilpotent ideal is Abelian if and only if there is a unique maximal Abelian ideal. (Although this is not used in the sequel, we note that this happens only for $\mathbf{C}_{n}$ and $\mathbf{G}_{2}$.)

Lemma 4.3. $\mathbb{H}_{\gamma, 1}$ meets $2 \mathbb{A}$ if and only if $\gamma$ is commutative.

\section{Proof:}

1. Suppose that $\gamma$ is not commutative. Then there exist $v_{1}, v_{2} \succcurlyeq \gamma$ such that $v_{1}+v_{2}=\theta$. If $x \in \mathbb{C} \cap \mathbb{H}_{\gamma, 1}$, then $\left(x, v_{i}\right) \geqslant 1$. Hence $(x, \theta) \geqslant 2$, i.e., $x \notin 2 \mathbb{A}$.

2. Suppose $\gamma$ is commutative, and let $w \in \hat{W}_{m c}$ be the minimal element of $\mathfrak{a}(\gamma)$. Then $w^{-1} *$ $\mathbb{A} \subset 2 \mathbb{A}$ and $H_{\gamma, 1}$ is the wall separating $w^{-1} * \mathbb{A}$ from the origin.

Next, we list some properties of (non)-commutative roots in $\Delta^{+}$. It would be very interesting to find an a priori proof.

\section{Theorem 4.4.}

(i) If $\theta=\sum_{i=1}^{n} c_{i} \alpha_{i}$, then $[\theta / 2]:=\sum_{i=1}^{n}\left[c_{i} / 2\right] \alpha_{i}$ is the unique maximal non-commutative root. (In case of $\mathfrak{s l}_{n+1}$, we temporarily assume that 0 is a root.)

(ii) (a) If the Dynkin diagram of $\Delta$ has no branching nodes, then the number of commutative roots is $n(n+1) / 2$.

(b) If there is a branching node and $n_{1}, n_{2}, n_{3}$ are the lengths of tails of the diagram obtained after deleting the branching node, hence $n_{1}+n_{2}+n_{3}=n-1$, then the number of commutative roots is $n(n+1) / 2+n_{1} n_{2} n_{3}$.

\section{Proof:}

(i) Suppose $\gamma=\sum_{i=1}^{n} m_{i} \alpha_{i} \in \Delta^{+}$and $m_{i}>\left[c_{i} / 2\right]$ for some $i$. Then $\gamma$ is obviously commutative. Hence all non-commutative roots satisfy the constraint $m_{i} \leqslant\left[c_{i} / 2\right]$ for all $i$. Then one observes a mysterious fact that both $[\theta / 2]$ and $\theta-[\theta / 2]$ are always roots. Since $[\theta / 2] \preccurlyeq \theta-[\theta / 2]$, we see that $[\theta / 2]$ is non-commutative.

(ii) (1) For $\mathfrak{g}=\mathfrak{s l}_{n+1}$, all positive roots are commutative.

(2) For $\mathfrak{g}=\mathfrak{s p}_{2 n}$, the commutative roots are $\left\{\varepsilon_{i}+\varepsilon_{j} \mid 1 \leqslant i \leqslant j \leqslant n\right\}$.

(3) For $\mathfrak{g}=\mathfrak{s o}_{2 n+1}$, the commutative roots are $\left\{\varepsilon_{i}+\varepsilon_{j} \mid 1 \leqslant i<j \leqslant n\right\} \cup\left\{\varepsilon_{1}-\varepsilon_{i} \mid\right.$ $2 \leqslant i \leqslant n\} \cup\left\{\varepsilon_{1}\right\}$.

(4) For $\mathfrak{g}=\mathfrak{s o}_{2 n}$, the commutative roots are $\left\{\varepsilon_{i}+\varepsilon_{j} \mid 1 \leqslant i<j \leqslant n\right\} \cup\left\{\varepsilon_{1}-\varepsilon_{i} \mid 2 \leqslant\right.$ $i \leqslant n\} \cup\left\{\varepsilon_{i}-\varepsilon_{n} \mid 2 \leqslant i \leqslant n-1\right\}$. 
(5) For the exceptional Lie algebras, one can perform explicit verification. E.g., the number of commutative roots is equal to $25,34,44$ for $\mathbf{E}_{6}, \mathbf{E}_{7}, \mathbf{E}_{8}$, respectively.

\section{Remark.}

1. For each $\gamma \in \Delta^{+}$, it is true that $[\gamma / 2] \in \Delta^{+} \cup\{0\}$ and $\gamma-[\gamma / 2] \in \Delta^{+}$.

2. Comparing Lemma 4.3 and Theorem 4.4(ii) shows that the number of hyperplanes that is needed to cut $2 \mathbb{A}$ into $2^{n}$ congruent simplices depends not only on $n$, but also on the angles between walls of $\mathbb{A}$.

In the Abelian case, the relationship between minimal elements and ad-nilpotent ideals works much better. This is explained by the fact that for $\mathfrak{c} \in \mathfrak{A} \mathfrak{d}$ we have $\ell\left(w_{\mathfrak{c}}\right)=\operatorname{dim} \mathfrak{c}$ if and only if $\mathfrak{c} \in \mathfrak{A} \mathfrak{b}$. For, it is known in general that $\ell\left(w_{\mathfrak{c}}\right)=\operatorname{dim} \mathfrak{c}+\operatorname{dim}[\mathfrak{c}, \mathfrak{c}]+\operatorname{dim}[\mathfrak{c},[\mathfrak{c}, \mathfrak{c}]]+\cdots$, see [4].

Suppose that $w \in \hat{W}_{m c}$ and $w^{-1}(\alpha)<0$ for $\alpha \in \hat{\Pi}$. Then the element $w^{\prime}=s_{\alpha} w$ whose length is one less is again minuscule. Conversely, if $w^{\prime} \in \hat{W}_{m c}$ and $w^{\prime}(\delta-\gamma)=\alpha \in \hat{\Pi}$ for some $\gamma \in \Delta^{+}$, then $w=s_{\alpha} w^{\prime}$ is also minuscule and $I_{w}=I_{w^{\prime}} \cup\{\gamma\}$ is the set of roots of an Abelian ideal [10, Theorem 2.4]. The procedure of such elementary extensions of Abelian ideals was studied in [10]. Properties of elementary extensions have useful consequences for describing the types of edges in $\mathbb{H}(\mathfrak{A} \mathfrak{b})$. The situation here resembles that in Section 1, i.e., the number of edges of any given type depends only on the length of the respective affine simple root.

Let us look again at Definition 3.6 (of the type of an edge). If $\gamma \in \Gamma(\mathfrak{c})$ and $I_{\mathfrak{c}^{\prime}}:=I_{\mathfrak{c}} \backslash\{\gamma\}$, then $w_{\mathfrak{c}}(\gamma-\delta)=: \alpha$ is the type of $\left(\mathfrak{c}, \mathfrak{c}^{\prime}\right)$. In this situation, we say that the generator $\gamma$ is of class $\alpha \in \hat{\Pi}$ in the ideal $\mathfrak{c}$. This notion is not, however, very convenient in general, since $\gamma$ can have another class in another ad-nilpotent ideal in which it is a generator. This is already seen in case $\mathfrak{g}=\mathfrak{s l}_{4}$, see Figure 2. But in the Abelian case this unpleasant phenomenon does not occur.

Theorem 4.5. Let $\gamma$ be a commutative root. Then the class of $\gamma$ does not depend on an Abelian ideal in which $\gamma$ is a generator.

Proof: Recall that $\mathfrak{a}(\gamma)$ is the minimal (Abelian) ad-nilpotent ideal generated by $\gamma$. All other Abelian ideals are obtained from $\mathfrak{a}(\gamma)$ via sequences of elementary extensions. So, it is enough to prove that if $\mathfrak{a}^{\prime} \subset \mathfrak{a}$ are Abelian, $\operatorname{dim} \mathfrak{a}^{\prime}=\operatorname{dim} \mathfrak{a}-1$, and $\gamma \in \Gamma(\mathfrak{a}) \cap \Gamma\left(\mathfrak{a}^{\prime}\right)$, then the classes of $\gamma$ in $\mathfrak{a}$ and $\mathfrak{a}^{\prime}$ are equal.

We have $I_{\mathfrak{a}}=I_{\mathfrak{a}^{\prime}} \cup\{\mu\}$ for some $\mu$. Since $\mu$ is a generator of $\mathfrak{a}$, we have $w_{\mathfrak{a}}(\mu-\delta)=\alpha_{i} \in$ $\hat{\Pi}$ and $w_{\mathfrak{a}}=s_{i} w_{\mathfrak{a}^{\prime}}$. Similarly, $w_{\mathfrak{a}}(\gamma-\delta)=\alpha_{j} \in \hat{\Pi}$. As $\gamma$ remains a generator of $\mathfrak{a}^{\prime}$, we have $\gamma \npreceq \mu$ and $\mu \nprec \gamma$. In particular, $(\gamma, \mu)=0$. Hence $\left(\alpha_{i}, \alpha_{j}\right)=(\mu-\delta, \gamma-\delta)=(\mu, \gamma)=0$. Therefore

$$
w_{\mathfrak{a}^{\prime}}(\gamma-\delta)=s_{i} w_{\mathfrak{a}}(\gamma-\delta)=s_{i}\left(\alpha_{j}\right)=\alpha_{j}=w_{\mathfrak{a}}(\gamma-\delta) .
$$

Thus, for any commutative root, the notion of class is well-defined. We write $\operatorname{cl}(\gamma)$ for the class of $\gamma$. The class can be regarded as the map $c l:\{$ commutative roots $\} \rightarrow \hat{\Pi}$. One can use the following strategy for computing the cardinality of $\mathcal{E}(\mathfrak{A} \mathfrak{b})_{i}$ : 
- Determine the class of each commutative root;

- If $c l^{-1}\left(\alpha_{i}\right)=:\left\{\mu_{1}, \ldots, \mu_{t}\right\}$, then for each $\mu_{j}$ one counts the number of Abelian ideals having $\mu_{j}$ as a generator. The total number of such ideals equals $\# \mathcal{E}(\mathfrak{A} \mathfrak{b})_{i}$.

The following lemma is helpful for explicit computations of the class.

\section{Lemma 4.6.}

(1) Let $\gamma, \gamma^{\prime}$ be commutative roots that are adjacent in $\mathbb{H}\left(\Delta^{+}\right)$. Then $\operatorname{cl}(\gamma), \operatorname{cl}\left(\gamma^{\prime}\right)$ are adjacent roots in the extended Dynkin diagram.

(2) Let $\gamma$ be a maximal root in the set $\left\{\mu \in \Delta^{+} \mid(\mu, \theta)=0\right\}$. Then $\operatorname{cl}(\gamma)=\alpha_{0}$.

(3) If $\gamma_{1}, \gamma_{2} \in \Gamma(\mathfrak{a})$ for some $\mathfrak{a} \in \mathfrak{A} \mathfrak{b}$, then $\left(\operatorname{cl}\left(\gamma_{1}\right), \operatorname{cl}\left(\gamma_{2}\right)\right)=0$.

\section{Proof:}

(1) Assume that $\gamma \preccurlyeq \gamma^{\prime}$. Let $\mathfrak{a}=\mathfrak{a}(\gamma)$ be the Abelian ideals generated by $\gamma$. Then $\gamma^{\prime} \in$ $\Gamma\left(I_{\mathfrak{a}} \backslash\{\gamma\}\right)$. Suppose that $w_{\mathfrak{a}}(\gamma-\delta)=\alpha \in \hat{\Pi}$, i.e., $c l(\gamma)=\alpha$. As follows from the above discussion on elementary extensions of Abelian ideals, $w^{\prime}=s_{\alpha} w_{\mathfrak{a}}$ is the minimal element of the upper ideal $I_{\mathfrak{a}} \backslash\{\gamma\}$. Hence, by Theorem 4.5, $\operatorname{cl}\left(\gamma^{\prime}\right)=w^{\prime}\left(\gamma^{\prime}-\delta\right)=: \alpha^{\prime}$. That is, $w_{\mathfrak{a}}\left(\gamma^{\prime}-\delta\right)=s_{\alpha}\left(\alpha^{\prime}\right)$. Since $\gamma^{\prime} \notin \Gamma(\mathfrak{a})$, we obtain $s_{\alpha}\left(\alpha^{\prime}\right) \notin \hat{\Pi}$. Hence $\left(\alpha, \alpha^{\prime}\right) \neq 0$.

(2) Let $I$ be the set of roots of $\mathfrak{a}(\gamma)$, and set $I^{\prime}=I \backslash\{\gamma\}$. Then $I^{\prime}$ is the set of roots of an Abelian ideal $\mathfrak{a}^{\prime}$ and $(\mu, \theta)>0$ for each $\mu \in I^{\prime}$. By Theorem 4.3 in [10], we then have $w_{\mathfrak{a}^{\prime}}=w s_{0}$ for some $w \in W$. Since $I^{\prime} \rightarrow I$ is an elementary extension of Abelian ideals, we must have $w_{\mathfrak{a}^{\prime}}(\delta-\gamma) \in \hat{\Pi}$. By the assumption, $w_{\mathfrak{a}^{\prime}}(\delta-\gamma)=\delta-w(\gamma)$. Hence the only possibility for this to be a simple root is $\alpha_{0}$. Thus $w_{\mathfrak{a}}=s_{0} w_{\mathfrak{a}^{\prime}}$.

(3) Suppose $w_{\mathfrak{a}}=s_{1} w^{\prime}=s_{2} w^{\prime \prime}$, where $\ell\left(w^{\prime}\right)=\ell\left(w^{\prime \prime}\right)=\ell(w)-1$, and $w_{\mathfrak{a}}\left(\delta-\gamma_{i}\right)=-\alpha_{i}$, $i=1,2$. If $\left(\alpha_{1}, \alpha_{2}\right) \neq 0$, then $\alpha_{1}+\alpha_{2} \in \hat{\Delta}^{+}$. Hence $w_{\mathfrak{a}}^{-1}\left(\alpha_{1}+\alpha_{2}\right)=\gamma_{1}+\gamma_{2}-2 \delta$. This means that $\gamma_{1}+\gamma_{2}$ is a root, i.e., $\mathfrak{a}$ is not Abelian.

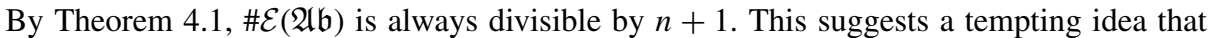
it might be true that the number of edges of each type equals $2^{n-2}$. But, one immediately finds that for $\mathfrak{s p}_{2 n}$ this is not the case. However, this is the only exception. Our results on the numbers $\# \mathcal{E}(\mathfrak{A} \mathfrak{b})_{i}$ are obtained via case-by-case considerations. To this end, one has to know the class of each commutative root. For $\mathfrak{s l}_{n+1}$, such an information is essentially presented in [10, Section 6.1] under the name of "filling the Ferrers diagram". Similar information for the other classical series is easily obtained via direct computations, using Proposition 3.10 and Lemma 4.6.

Theorem 4.7. If $\mathfrak{g}=\mathfrak{s l}_{n+1}, n \geqslant 2$, then $\# \mathcal{E}(\mathfrak{A} \mathfrak{b})_{i}=2^{n-2}$ for each $i \in\{0,1, \ldots, n\}$.

Proof: In $\mathfrak{s l}_{n+1}$, the positive (= commutative) roots are $v_{i j}:=\varepsilon_{i}-\varepsilon_{j}, 1 \leqslant i<j \leqslant n+1$. The numbering of simple roots is such that $\alpha_{i}=v_{i, i+1}, i=1, \ldots, n$. Then $\operatorname{cl}\left(v_{i j}\right)=\alpha_{k}$, where $i+j-1 \equiv k(\bmod n+1)$. We work with the usual matrix realisation of $\mathfrak{b}$ as the set of upper-triangular matrices. The set of positive roots is identified with the right-justified Ferrers diagram with row lengths $(n, n-1, \ldots, 1)$, and the Abelian upper ideals are identified with the right-justified subdiagrams of it that fit inside the rectangle of shape $j \times(n+1-j)$ for some $j$. Such subdiagrams are said to be Abelian Ferrers diagrams. The class of roots is constant along the diagonals parallel to the antidiagonal (in the $n+1$ by $n+1$ matrix). In particular, the roots of class $\alpha_{0}$ are exactly the roots on the antidiagonal, see Figure 3 . 


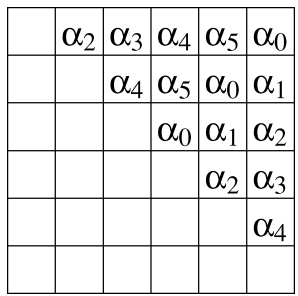

Fig. 3 Classes of the commutative roots for $\mathfrak{s l}_{6}$

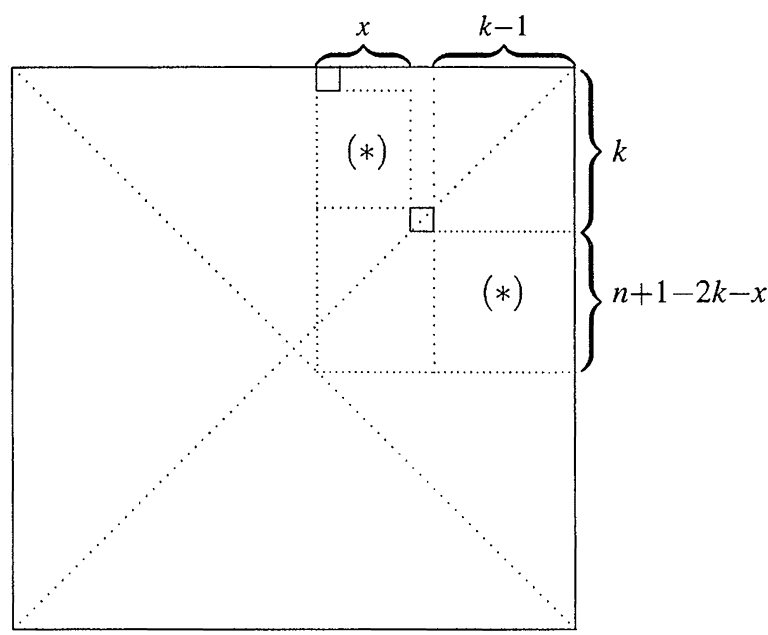

Fig. 4

The automorphism group of $\mathbb{H}\left(\mathfrak{A b}\left(\mathfrak{s l}_{n+1}\right)\right)$ (as undirected graph!) is equal to $D_{n+1}$, the dihedral group of order $2(n+1)$, see [19]. Furthermore, Suter explicitly describes a certain automorphism $\tau$ of order $n+1$, via a "sliding procedure in south-east direction". Comparing that procedure with the formula for the class of roots, and thereby the types of edges in $\mathcal{E}(\mathfrak{A} \mathfrak{b})$, one finds that $\tau$ always takes an edge of type $\alpha_{i}$ in $\mathcal{E}(\mathfrak{A} \mathfrak{b})$ to an edge of type $\alpha_{i+2}$ (with the cyclic ordering of affine simple roots), see Lemma 4.8 below. Hence, if $n+1$ odd, then $\tau$ acts transitively on the set of types of edges, which completes the proof in this case. If $n+1$ is even, then $\tau$ has 2 orbits on the set of types, i.e., $\{0,2,4, \ldots, n-1\}$ and $\{1,3, \ldots, n\}$. Therefore it is enough to check that the number of Abelian ideals having a generator of class $\alpha_{0}$ equals $2^{n-2}$. Note that the verification performed below does not exploit the hypothesis that $n+1$ is even.

Take the commutative root of class $\alpha_{0}$ that lies in the $k$-th row of the Ferrers diagram (matrix), i.e., $v_{k, n+2-k}$. Then $k \leqslant(n+1) / 2$. Consider the set of Abelian Ferrers diagram having this root as a generator (= south-west corner) and the first row of length $x+k$. Using the graphical presentation of ideals, see Figure 4, we obtain that the cardinality of this set equals

$$
\left\{\begin{array}{l}
\text { the number of all Ferrers diagrams } \\
\text { that fit in the rectangle of shape } \\
x \times(k-2)
\end{array}\right\} \times\left\{\begin{array}{l}
\text { the number of all Ferrers diagrams } \\
\text { that fit in the rectangle of shape } \\
(n+1-2 k-x) \times(k-1)
\end{array}\right\} .
$$


(Two rectangles in Figure 4 that have to be filled with Ferrers diagrams are marked with (*).) Here the first (resp. second) number equals $\left(\begin{array}{c}x+k-2 \\ k-2\end{array}\right)$ (resp. $\left(\begin{array}{c}n-k-x \\ k-1\end{array}\right)$ ). Therefore the total number of Abelian ideals having the generator of class $\alpha_{0}$ lying in the $k$-th row is equal to

$$
\sum_{x=0}^{n+1-2 k}\left(\begin{array}{c}
x+k-2 \\
k-2
\end{array}\right)\left(\begin{array}{c}
n-k-x \\
k-1
\end{array}\right)
$$

Using Lemma 4.9 below, we obtain this sum equals $\left(\begin{array}{c}n-1 \\ 2 k-2\end{array}\right)$. Hence the total number of Abelian ideals having a generator of class $\alpha_{0}$ is equal to

$$
\sum_{k=1}^{(n+1) / 2}\left(\begin{array}{c}
n-1 \\
2 k-2
\end{array}\right)=2^{n-2}
$$

Now we prove two results that have been used in the previous proof.

Lemma 4.8. Let $\tau$ be Suter's automorphism of the undirected graph $\mathbb{H}\left(\mathfrak{A b}\left(\mathfrak{s l}_{n+1}\right)\right)$ (to be defined below). Then $\tau$ takes an edge of type $\alpha_{i}$ to an edge of type $\alpha_{i+2}$, with the cyclic ordering of the affine simple roots.

Proof: In this proof, we write $(a, b)$ for the root $v_{a b}$. Let $\mathfrak{a}$ be the Abelian ideal with generators $\Gamma(\mathfrak{a})=\left\{\left(a_{1}, b_{1}\right), \ldots,\left(a_{k}, b_{k}\right)\right\}$. Then $1 \leqslant a_{1}<\ldots<a_{k}<b_{1}<\ldots<b_{k} \leqslant n+1$. The generators of $\tau(\mathfrak{a})$ are defined by the following rule (it is a formal version of the diagrams depicted in [19]):

- If $b_{k}=n+1$, then $\left(a_{k}, b_{k}\right)$ disappears. In all other cases, $\left(a_{j}, b_{j}\right)$ is replaced with $\left(a_{j}+\right.$ $\left.1, b_{j}+1\right)$

- If $a_{k}+1<b_{1}$, then the new generator $\left(1, a_{k}+2\right)$ emerges.

Now, we have to keep track of all edges incident to $\mathfrak{a}$, not only those terminating in $\mathfrak{a}$. The edges terminating in $\mathfrak{a}$ correspond to the generators, i.e., the south-west corners of the respective Ferrers diagram, whereas the edges originating in $\mathfrak{a}$ correspond to the maximal roots in $\Delta^{+} \backslash I_{\mathfrak{a}}$ (modulo the constraint that $\mathfrak{a}$ plus the respective root space still yields an Abelian ideal). Hence, the full collection of edges incident to $\mathfrak{a}$ is determined by the roots: terminating in $\mathfrak{a}:\left(a_{1}, b_{1}\right), \ldots,\left(a_{k}, b_{k}\right)$; originating in $\mathfrak{a}$ : $\left(a_{1}+1, b_{2}-1\right), \ldots,\left(a_{k-1}+1, b_{k}-1\right)$; furthermore, if $a_{k}+1<b_{1}$, It is easy to see how then the roots $\left(1, b_{1}-1\right),\left(a_{k}+1, n+1\right)$ are also needed.

these roots transform under $\tau$. If a root $(a, b)$ does not belong to the last column of the matrix, then it merely goes to $(a+1, b+1)$. Hence the "increment" in the type number is 2 . All possibilities for the roots lying in the last column are easily handled in a case-by-case fashion

Lemma 4.9. For $a, b, c \in \mathbb{N}$ and $a \geqslant b+c$, we have

$$
\sum_{x=c}^{a-b}\left(\begin{array}{l}
x \\
c
\end{array}\right)\left(\begin{array}{c}
a-x \\
b
\end{array}\right)=\left(\begin{array}{c}
a+1 \\
b+c+1
\end{array}\right) .
$$


Proof: Denoting the left-hand side by $F(a, b, c)$ and using the equality $\left(\begin{array}{l}x \\ c\end{array}\right)=\sum_{y=c}^{x}\left(\begin{array}{l}y-1 \\ c-1\end{array}\right)$, we obtain

$$
\begin{aligned}
F(a, b, c) & =\sum_{x=c}^{a-b}\left(\begin{array}{c}
a-x \\
b
\end{array}\right) \sum_{y=c}^{x}\left(\begin{array}{c}
y-1 \\
c-1
\end{array}\right)=\sum_{y=c}^{a-b}\left(\begin{array}{c}
y-1 \\
c-1
\end{array}\right) \sum_{x=y}^{a-b}\left(\begin{array}{c}
a-x \\
b
\end{array}\right) \\
& =\cdots=F(a, b+1, c-1) .
\end{aligned}
$$

Hence, it suffices to treat the case $c=0$, which is easy.

For $\mathfrak{s p}_{2 n}$, the automorphism group of $\mathbb{H}\left(\mathfrak{A} \mathfrak{b}\left(\mathfrak{s p}_{2 n}\right)\right)$ is not as rich as for $\mathfrak{s l}_{n+1}$. But a direct computation is not difficult.

Theorem 4.10. If $\mathfrak{g}=\mathfrak{s p}_{2 n}, \quad n \geqslant 1$, then $\# \mathcal{E}(\mathfrak{A} \mathfrak{b})_{i}=2^{n-2}$ for $i \in\{1, \ldots, n-1\}$ and $\# \mathcal{E}(\mathfrak{A} \mathfrak{b})_{0}=2^{n-1}$.

Proof: In $\mathfrak{s p}_{2 n}$, the commutative roots are $\gamma_{i j}:=\varepsilon_{i}+\varepsilon_{j}, 1 \leqslant i \leqslant j \leqslant n$ and $c l\left(\gamma_{i j}\right)=\alpha_{j-i}$. This already shows that there is no commutative roots of class $\alpha_{n}$, and thereby no edges of type $\alpha_{n}$ in $\mathcal{E}(\mathfrak{A} \mathfrak{b})$.

The standard matrix realisation of $\mathfrak{s p}_{2 n}$ shows that the set of commutative roots is identified with the left-justified Ferrers diagram with row lengths $(n, n-1, \ldots, 1)$, and the Abelian upper ideals are identified with shifted Ferrers subdiagrams inside, cf. also [11, (5.1)]. For instance, the 7-dimensional ideal in the right diagram in Figure 5 has the generators $\gamma_{14}$ and $\gamma_{33}$ of classes $\alpha_{0}$ and $\alpha_{3}$, respectively. For brevity, we will say that an Abelian ideal in $\mathfrak{s p}_{2 n}$ is depicted by a triangular Ferrers diagram of size $\leqslant n$. Take the commutative root of class $\alpha_{i}$ lying in the $k$-th row of the diagram, i.e., $\gamma_{i, i+k}$. Then $k \leqslant n-i$. Using these pictures, we find that the number of triangular Ferrers diagram of size $\leqslant n$ having this root as a generator (= south-west corner) equals

$$
\left\{\begin{array}{l}
\text { the number of the (usual) Ferrers diagrams } \\
\text { that fit in the rectangle of the shape }(n- \\
i-k) \times(k-1)
\end{array}\right\} \times\left\{\begin{array}{l}
\text { the number of triangular Ferrers di- } \\
\text { agrams of size } \leqslant i-1
\end{array}\right\}
$$

Here the first number equals $\left(\begin{array}{c}n-i-1 \\ k-1\end{array}\right)$, and the second one equals $2^{i-1}$, if $i \geqslant 1$. Therefore the total number of Abelian ideals having a generator of class $\alpha_{i}$ is equal to

$$
\begin{aligned}
& \sum_{k=1}^{n-i}\left(\begin{array}{c}
n-i-1 \\
k-1
\end{array}\right) 2^{i-1}=2^{n-i-1} 2^{i-1}=2^{n-2}
\end{aligned}
$$

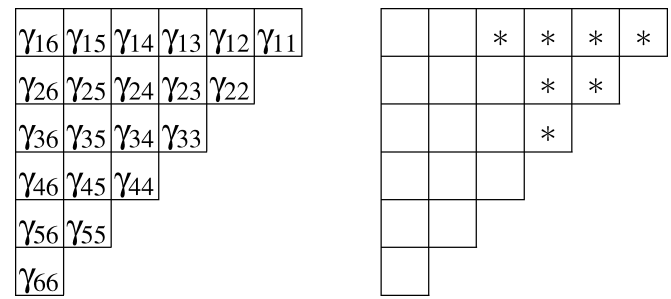

Fig. 5 The commutative roots and an Abelian ideal for $\mathfrak{s p}_{12}$ 


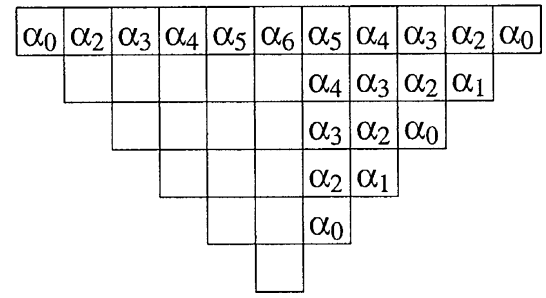

Fig. 6 The classes of commutative roots for $\mathfrak{s o}_{13}$

Similarly, for $i=0$ we obtain

$$
\sum_{k=1}^{n}\left(\begin{array}{l}
n-1 \\
k-1
\end{array}\right)=2^{n-1}
$$

Theorem 4.11. If $\mathfrak{g}=\mathfrak{s o}_{2 n+1}$ or $\mathfrak{s o}_{2 n}, \quad n \geqslant 3$, then $\# \mathcal{E}(\mathfrak{A} \mathfrak{b})_{i}=2^{n-2}$ for each $i \in$ $\{0,1, \ldots, n\}$.

Proof: (1) $\mathfrak{g}=\mathfrak{s o}_{2 n+1}$. The classes of the commutative roots are as follows:

$\operatorname{cl}\left(\varepsilon_{1}-\varepsilon_{2}\right)=\alpha_{0}, \operatorname{cl}\left(\varepsilon_{1}-\varepsilon_{j}\right)=\alpha_{j-1}$, where $3 \leqslant j \leqslant n+1$ and $\varepsilon_{n+1}:=0$, and

$$
c l\left(\varepsilon_{i}+\varepsilon_{j}\right)= \begin{cases}\alpha_{j-i}, & \text { if } j-i \geqslant 2, \\ \alpha_{0}, & \text { if } j-i=1 \text { and } j \text { is even, where } 1 \leqslant i<j \leqslant n . \\ \alpha_{1}, & \text { if } j-i=1 \text { and } j \text { is odd. }\end{cases}
$$

Notice that $\varepsilon_{1}$ is the only short commutative roots and hence the only root of class $\alpha_{n}$. The standard matrix realisation of $\mathfrak{s o}_{2 n+1}$ shows that the set (upper ideal) of commutative roots is identified with a skew Ferrers diagram, with row lengths $(2 n-1, n-2, n-3, \ldots, 1)$, see the sample Figure for $\mathfrak{s o}_{13}$, where the leftmost (resp. rightmost) box corresponds to $\alpha_{1}=\varepsilon_{1}-\varepsilon_{2}$ (resp. $\theta=\varepsilon_{1}+\varepsilon_{2}$ ) and the empty boxes represent the non-commutative roots.

For each commutative root, we can compute the number of Abelian ideals having this root as a generator. (It is important that the ideal of the commutative roots is not Abelian, so that one should count sub-ideals that are really Abelian.) For instance, if $i \geqslant 2$ and $j-i \geqslant 2$, then the number of Abelian ideals having $\varepsilon_{i}+\varepsilon_{j}$ as a generator is equal to $2^{j-i-1}\left(\begin{array}{c}n-j+i-1 \\ i-1\end{array}\right)$; if $i \geqslant 2$ and $j-i=1$, then the number of ideals having $\varepsilon_{i}+\varepsilon_{i+1}$ as a generator is equal to $2\left(\begin{array}{c}n-2 \\ i-1\end{array}\right)$. For the roots $\varepsilon_{1} \pm \varepsilon_{j}$, the computations and the answer are even easier. (We omit explicit manipulations with Ferrers diagrams.)

Then taking the sum of the numbers obtained corresponding to the roots of the same class yields the answer.

(2) $\mathfrak{g}=\mathfrak{s o}_{2 n}$. The classes of the commutative roots are as follows:

$\operatorname{cl}\left(\varepsilon_{1}-\varepsilon_{2}\right)=\alpha_{0}, \operatorname{cl}\left(\varepsilon_{1}-\varepsilon_{j}\right)=\alpha_{j-1}(3 \leqslant j \leqslant n-1), \operatorname{cl}\left(\varepsilon_{i}-\varepsilon_{n}\right)=\alpha_{n-i}(1 \leqslant i \leqslant n-2)$, $\operatorname{cl}\left(\varepsilon_{n-1}-\varepsilon_{n}\right)=\operatorname{cl}\left(\varepsilon_{n-1}+\varepsilon_{n}\right)$, and also equation (4.12).

Again, it is easier to understand this by looking at the sample picture for $\mathfrak{s o}_{12}$, which is associated with the standard matrix realisation of $\mathfrak{s o}_{2 n}$.

We omit all other details for this case. 


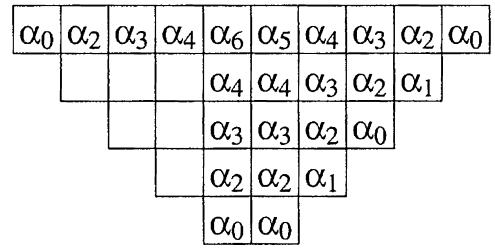

Fig. 7 The classes of commutative roots for $\mathfrak{s o}_{12}$

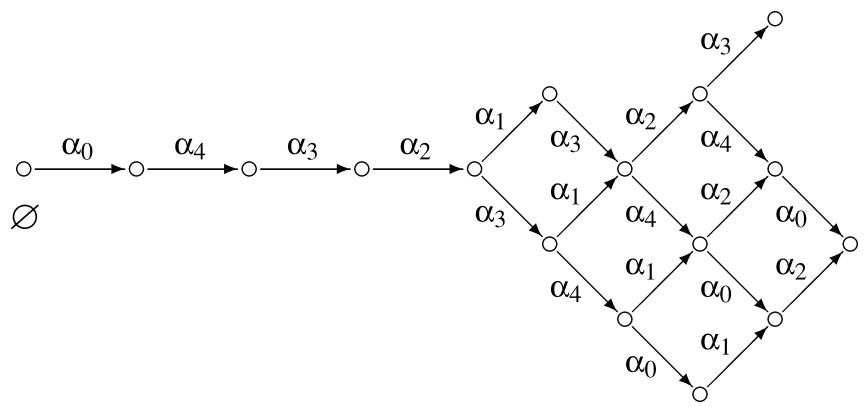

Fig. 8 The Hasse diagram of $\mathfrak{A} \mathfrak{b}\left(\mathbf{F}_{4}\right)$, with types of edges

Theorems 4.7, 4.10, 4.11, and our computations for the exceptional Lie algebras prove the following general result.

Theorem 4.13. For each simple Lie algebra $\mathfrak{g}$, the numbers $\# \mathcal{E}(\mathfrak{A} \mathfrak{b})_{i}$ depend only on the length of $\alpha_{i}$. Moreover, if $\mathfrak{g} \neq \mathfrak{s p}_{2 n}$, then the number of edges of each type in $\mathbb{H}(\mathfrak{A} \mathfrak{b})$ is equal to $2^{n-2}$ (Warning: $\mathfrak{s l}_{2}$ is regarded as $\mathfrak{s p}_{2}$ ).

Our proof for the exceptional Lie algebras uses the same strategy together with the explicit knowledge of classes of the commutative roots. The classes of all commutative roots for $\mathbf{E}_{6}, \mathbf{E}_{7}, \mathbf{E}_{8}, \mathbf{F}_{4}$ are indicated in the Appendix. The case of $\mathbf{G}_{2}$ is trivial. In the figure for $\mathbf{F}_{4}$, the leftmost node corresponds to the zero-dimensional ideal and the two sinks on the right represent two maximal Abelian ideals.

Remark 4.14. If $\Delta$ is not simply-laced, then one may consider the long Abelian ideals, i.e., the Abelian ideals such that $I_{\mathfrak{a}}$ contains only long roots [12]. The corresponding poset, denoted $\mathfrak{A b}_{l}$, consists of 2, 3, 4 elements for $\mathbf{C}_{n}, \mathbf{G}_{2}, \mathbf{F}_{4}$, respectively. The only interesting case is that of $\mathbf{B}_{n}$, where $\# \mathfrak{A} \mathfrak{b}_{l}=2^{n-1}$. It follows from [12, Prop. 2.1] that the type of any edge of $\mathbb{H}\left(\mathfrak{A} \mathfrak{b}_{l}\right)$ is a long root from $\hat{\Pi}$. One can show here that the number of edges of each

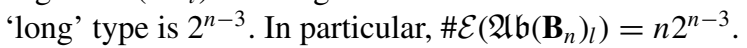

\section{The covering polynomial of a finite poset}

Results on counting edges, especially Proposition 3.3, suggest a general construction of a polynomial attached to a finite poset.

Let $(\mathcal{P}, \preccurlyeq)$ be a finite poset. Write $\mathcal{E}(\mathcal{P})$ for the set of edges of the Hasse diagram of $\mathcal{P}$. We define a polynomial which encodes some properties of the covering relation in $\mathcal{P}$. For any $x \in \mathcal{P}$, let $\kappa(x)$ be the number of $y \in \mathcal{P}$ such that $y$ is covered by $x$. 
Definition 5.1. The covering polynomial of $\mathcal{P}$ is

$$
\mathcal{K}_{\mathcal{P}}(q)=\sum_{x \in \mathcal{P}} q^{\kappa(x)}
$$

It follows from the definition that

$$
\left.\mathcal{K}_{\mathcal{P}}(q)\right|_{q=1}=\# \mathcal{P} \quad \text { and }\left.\quad \frac{d}{d q} \mathcal{K}_{\mathcal{P}}(q)\right|_{q=1}=\# \mathcal{E}(\mathcal{P}) .
$$

On the other hand, $\mathcal{K}_{\mathcal{P}}(0)$ is the number of the minimal elements in $\mathcal{P}$. It might be interesting to realise whether some other values of $\mathcal{K}_{\mathcal{P}}$ have a combinatorial meaning.

Example. For the Boolean lattice $\mathcal{B}_{n}$, we have $\mathcal{K}_{\mathcal{B}_{n}}(q)=(1+q)^{n}$.

Let us look at the covering polynomials of posets considered above.

(A) It is not hard to compute the covering polynomial for the posets $\Delta^{+}$. Here is the answer:

\begin{tabular}{cl}
\hline$\Delta$ & \multicolumn{1}{c}{$\mathcal{K}_{\Delta^{+}}(q)$} \\
\hline $\mathbf{A}_{n}$ & $n+\left(\begin{array}{c}n \\
2\end{array}\right) q^{2}$ \\
$\mathbf{B}_{n}, \mathbf{C}_{n}$ & $n+(n-1) q+(n-1)^{2} q^{2}$ \\
$\mathbf{D}_{n}$ & $n+(n-3) q+\left(\left(\begin{array}{c}n \\
2\end{array}\right)+\left(\begin{array}{c}n-3 \\
2\end{array}\right)\right) q^{2}+(n-3) q^{3}$ \\
$\mathbf{E}_{6}$ & $6+5 q+20 q^{2}+5 q^{3}$ \\
$\mathbf{E}_{7}$ & $7+10 q+36 q^{2}+10 q^{3}$ \\
$\mathbf{E}_{8}$ & $8+21 q+70 q^{2}+21 q^{3}$ \\
$\mathbf{F}_{4}$ & $4+7 q+12 q^{2}+q^{3}$ \\
$\mathbf{G}_{2}$ & $2+3 q+q^{2}$ \\
\hline
\end{tabular}

Notice that (1) $\operatorname{deg} \mathcal{K}_{\Delta^{+}} \leqslant 3$, and (2) if $\Delta$ is simply-laced, then the coefficients of $q$ and $q^{3}$ are equal. The first claim can be proved a priori; the second claim is derived from the first one using the equalities $\mathcal{K}_{\Delta^{+}}(1)=n h / 2$ and $\mathcal{K}_{\Delta^{+}}^{\prime}(1)=n(h-2)$, where the last equality stems from Theorem 1.1.

(B) If $\mathcal{P}=\mathfrak{A} \mathfrak{d}(\mathfrak{g})$, then $\kappa(\mathfrak{c})$ is the number of generators of $\mathfrak{c}$ for any $\mathfrak{c} \in \mathfrak{A} \mathfrak{d}(\mathfrak{g})$. Hence the covering polynomial of $\mathfrak{A} \mathfrak{d}(\mathfrak{g})$ is nothing but the generalised Narayana polynomial $\mathcal{N}_{\mathfrak{g}}(q)$ from Section 3. These polynomials are explicitly written out in [11, Section 6]. See also [16, Section 5.2] and [1,5.2], where $\mathcal{N}_{\mathfrak{g}}(q)$ appears as the $h$-polynomial of the polytope $\mathcal{M}(\mathfrak{g})$ mentioned in Remark 3.5 and a Poincaré polynomial for the set of simple elements in the dual braid monoid, respectively.

(C) More generally, if $\mathbb{X}$ is any class of ad-nilpotent ideals that is closed under taking sub-ideals, then $\kappa(\mathfrak{c})$ (for the corresponding poset) is just the number of generators of $\mathfrak{c}$. In particular, this applies to $\mathbb{X}=\mathfrak{A} \mathfrak{b}$.

The covering polynomial for $\mathcal{P}=\mathfrak{A} \mathfrak{b}$ is computed for each simple Lie algebra separately. We first note that the coefficient of $q$ is the number of commutative roots. It follows from a result of Sommers [17, Theorem 6.4] that the number of generators of an Abelian ideal is at most the maximal number of orthogonal roots in $\Pi$. This provides an upper bound on $\operatorname{deg} \mathcal{K}_{\mathfrak{A} \mathfrak{b}}$. Actually, it is easy to find out an Abelian ideal with such a number of generators, 且 Springer 
so that one obtains the precise value of the degree. For instance, deg $\mathcal{K}_{\mathfrak{A b}_{\left(\mathbf{D}_{n}\right)}}=\left[\frac{n}{2}\right]+1$, $\operatorname{deg} \mathcal{K}_{\mathfrak{A} \mathfrak{b}\left(\mathbf{E}_{7}\right)}=\operatorname{deg} \mathcal{K}_{\mathfrak{A} \mathfrak{b}\left(\mathbf{E}_{8)}\right)}=4$.

If $\operatorname{deg} \mathcal{K}_{\mathfrak{A b}} \leqslant 3$, then this polynomial can be computed using the values $\mathcal{K}_{\mathfrak{A b}}(1)=2^{n}$, $\mathcal{K}_{\mathfrak{A} \mathfrak{b}}^{\prime}(1)=(n+1) 2^{n-2}$, and the known number of commutative roots. The result for $\mathbf{A}_{n}$ and $\mathbf{C}_{n}$ can be deduced from matrix descriptions of generators given in [15, Section 3]. It is a mere coincidence that the covering polynomial for $\mathfrak{A b}\left(\mathbf{B}_{n}\right)$ is the same as for $\mathbf{A}_{n}$ and $\mathbf{C}_{n}$. The formulae for both orthogonal series result from direct matrix considerations, which are similar to that in Theorems 4.7, 4.11 and [15, Section 3]. The proof for $\mathbf{D}_{n}$ is more involved, but still it can be derived from the consideration of the Ferrers diagram for the ad-nilpotent ideal of commutative roots. (We came up with the explicit formula for $\mathbf{D}_{n}$ using computer calculations made by $\mathrm{G}$. Röhrle for $\mathbf{D}_{n}, n=7,8$.) The details of these computations and some related results on covering polynomials will appear elsewhere.

\begin{tabular}{cl}
\hline $\mathfrak{g}$ & \multicolumn{1}{c}{$\mathcal{K}_{\mathfrak{A} \mathfrak{b}}(q)$} \\
\hline $\mathbf{A}_{n}, \mathbf{B}_{n}, \mathbf{C}_{n}$ & $\sum_{k \geqslant 0}\left(\begin{array}{c}n+1 \\
2 k\end{array}\right) q^{k}$ \\
$\mathbf{D}_{n}$ & $\sum_{k \geqslant 0}\left(\left(\begin{array}{c}n+2 \\
2 k\end{array}\right)-4\left(\begin{array}{c}n-1 \\
2 k-2\end{array}\right)\right) q^{k}$ \\
$\mathbf{E}_{6}$ & $1+25 q+27 q^{2}+11 q^{3}$ \\
$\mathbf{E}_{7}$ & $1+34 q+60 q^{2}+30 q^{3}+3 q^{4}$ \\
$\mathbf{E}_{8}$ & $1+44 q+118 q^{2}+76 q^{3}+17 q^{4}$ \\
$\mathbf{F}_{4}$ & $1+10 q+5 q^{2}$ \\
$\mathbf{G}_{2}$ & $1+3 q$ \\
\hline
\end{tabular}

\section{Appendix A. Classes of the commutative roots for $\mathbf{E}_{6}, \mathbf{E}_{7}, \mathbf{E}_{8}, \mathbf{F}_{4}$}

We follow the natural numbering of simple roots, i.e., that adopted in [21]. To convey the idea of "naturality" to the interested reader, we present this graphically:

$$
\mathbf{E}_{6}: \begin{gathered}
1-2-3-4-5 \\
6
\end{gathered} \quad \mathbf{E}_{7}: \begin{gathered}
1-2-3-4-5-6 \\
7
\end{gathered} \quad \mathbf{E}_{8}: \begin{gathered}
1-2-3-4-5-6-7 \\
8
\end{gathered} \quad \mathbf{F}_{4}: \quad 1-2 \Leftarrow 3-4 .
$$

For brevity, we write $\left[m_{1} m_{2} \ldots m_{n}\right]$ in place of $\sum_{i=1}^{n} m_{i} \alpha_{i}$.

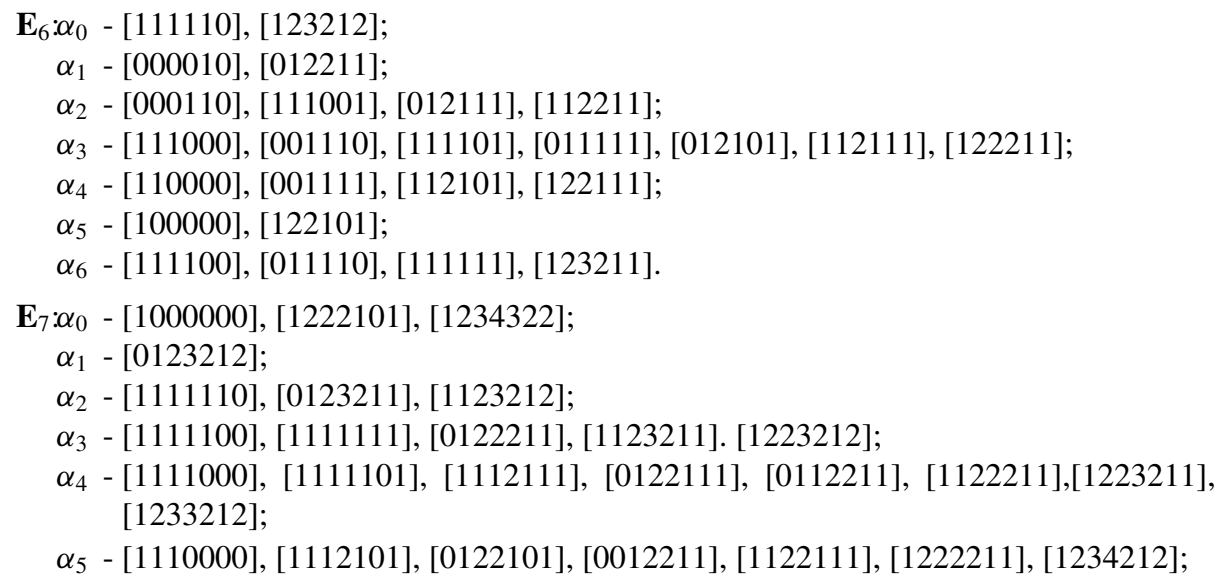




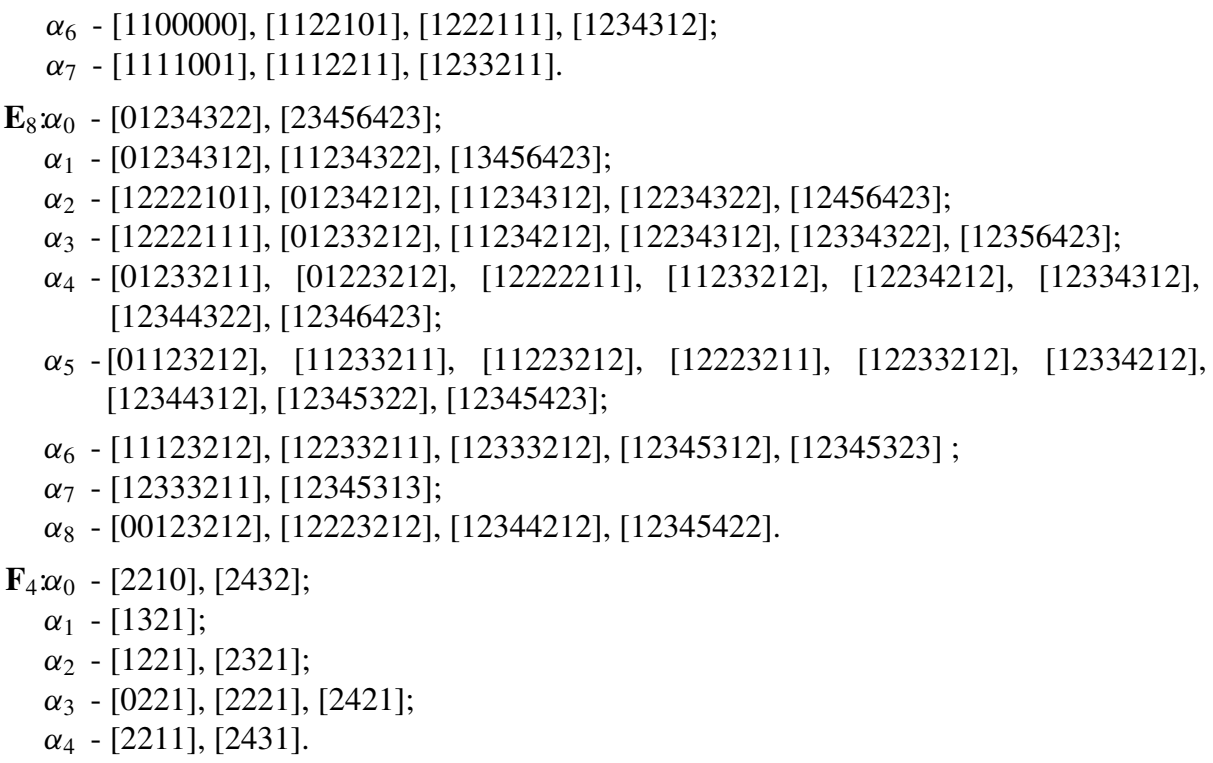

To obtain these lists, we combine explicit computations of certain minimal elements with using Proposition 3.10 and Lemma 4.6. The information for $\mathbf{F}_{4}$ is also readily being extracted from the data of Table 6.1 in [10].

Acknowledgments Part of this work was done while I was visiting the Max-Planck-Institut für Mathematik (Bonn). I thank the Institute for its hospitality and inspiring environment.

\section{References}

1. D. Bessis, "The dual braid monoid," Ann. Sci. École Norm. Sup., Sér. IV. 36 (2003), 647-683.

2. N. Bourbaki, "Groupes et algèbres de Lie," Chapitres 4,5 et 6, Paris: Hermann 1975.

3. R. Brylinski and B. Kostant, "Nilpotent orbits, normality, and Hamiltonian group actions," J. Amer. Math. Soc. 7 (1994), 269-298.

4. P. Cellini and P. Papi, "ad-nilpotent ideals of a Borel subalgebra," J. Algebra 225 (2000), 130-141.

5. P. Cellini and P. Papi, "ad-nilpotent ideals of a Borel subalgebra II," J. Algebra 258 (2002), 112-121.

6. P. Cellini and P. Papi, "Abelian ideals of Borel subalgebras and affine Weyl groups," Adv. Math. 187 (2004), 320-361.

7. F. Chapoton, S. Fomin, and A. Zelevinsky, "Polytopal realizations of generalized associahedra," Canad. Math. Bull. 45(4) (2002), 537-566.

8. J.E. Humphreys, Reflection Groups and Coxeter Groups, Cambridge Univ. Press, 1992.

9. B. Kostant, "The set of abelian ideals of a Borel subalgebra, Cartan decompositions, and discrete series representations," Intern. Math. Res. Notices, 5 (1998), 225-252.

10. D. Panyushev, "Abelian ideals of a Borel subalgebra and long positive roots," Intern. Math. Res. Notices 35 (2003), 1889-1913.

11. D. Panyushev, "Ad-nilpotent ideals of a Borel subalgebra: generators and duality," J. Algebra 274 (2004), 822-846.

12. D. Panyushev, "Long Abelian ideals," Adv. Math. 186 (2004), 307-316.

13. D. Panyushev, "Ideals of Heisenberg type and minimax elements of affine Weyl groups," in Amer. Math. Soc. Transl., Series 2. "Lie Groups and Invariant Theory" E.B.Vinberg, (Ed.), 213 (2005), 191-213. (=Preprint arXiv: math.RT/0311347, $25 \mathrm{pp}$ ).

14. D. Panyushev, "Normalizers of ad-nilpotent ideals," Europ. J. Combinatorics, 27 (2006), 153-178. (= Preprint arXiv: math.RT/0402140).

15. D. Panyushev and G. Röhrle, "Spherical orbits and Abelian ideals," Adv. Math. 159 (2001), 229-246. 
16. V. Reiner and V. Welker, “On the Charney-Davis and Neggers-Stanley Conjectures," J. Combin Theory, Ser. A 109 (2005) no. 2, 247-280. http: / /www. math. umn. edu/ reiner/Papers/papers.html, 2002.

17. E. Sommers, "B-stable ideals in the nilradical of a Borel subalgebra," Canad. Math. Bull., 48 (2005), no. 3, 460-472. (= Preprint arXiv: math.RT/0303182).

18. R. Suter Coxeter and dual Coxeter numbers, Comm. Alg. 26 (1998), 147-153.

19. R. Suter, "Young's lattice and dihedral symmetries," Europ. J. Combinatorics 23(2002), 233-238.

20. R. Suter, "Abelian ideals in a Borel subalgebra of a complex simple Lie algebra," Invent. Math. 156 (2004), $175-221$.

21. Москва: “Наука” 1988 (Russian). Э.Б. ВинБерг, А.Л. Онищик. Семинар по группам Ли и алгебраическим группам. Москва:English translation in A.L. Onishchik and E.B. Vinberg, "Lie groups and algebraic groups," Berlin: Springer, 1990. 